On the uniqueness, stability and hyperbolicity of symmetric and periodic solutions of weakly nonlinear ordinary differential equations

\author{
Dilna Nataliya and Michal Fečkan
}




\title{
ON THE UNIQUENESS, STABILITY AND HYPERBOLICITY OF SYMMETRIC AND PERIODIC SOLUTIONS OF WEAKLY NONLINEAR ORDINARY DIFFERENTIAL EQUATIONS
}

\author{
NATALIYA DILNA AND MICHAL FEČKAN
}

Received 12 November, 2008

\begin{abstract}
We show the existence of unique periodic and symmetric solutions of weakly nonlinear ordinary differential equations. Conditions of stability and hyperbolicity of these solutions are established as well. Concrete examples are presented to show advantages of our method to the classical averaging theory.
\end{abstract}

2000 Mathematics Subject Classification: 34C14, 34C15, 34C25

Keywords: periodic solution, symmetric systems, stability of solution, $k$-hyperbolicity

\section{INTRODUCTION}

In this paper we consider systems of ordinary differential equations under periodic and symmetric assumptions. More concretely, we mostly consider a weakly nonlinear ordinary differential equation of the form

$$
x^{\prime}(t)=\varepsilon f(x(t), t), \quad t \in \mathbb{R},
$$

with a small parameter $\varepsilon \in \mathbb{R}$, where $x: \mathbb{R} \rightarrow \mathbb{R}^{n}$. We suppose that the function $f: \mathbb{R}^{n+1} \rightarrow \mathbb{R}^{n}$ in (1.1) is sufficiently smooth, $p T$-periodic in the second variable, and symmetric in the first one, i. e.,

$$
A f(x, t)=f(A x, t+T), \quad t \in \mathbb{R},
$$

where $A: \mathbb{R}^{n} \rightarrow \mathbb{R}^{n}$ is a linear map such that $A^{p}=\mathbb{1}$ for some $p \in \mathbb{N}$. Due to this property, we have the following useful construction. Let $\langle\cdot, \cdot\rangle$ be a scalar product on $\mathbb{R}^{n}$. By setting

$$
(x, y):=\frac{1}{p} \sum_{i=0}^{p-1}\left\langle A^{i} x, A^{i} y\right\rangle, \quad\{x, y\} \subset \mathbb{R}^{n},
$$

The first author was supported in part by the National Scholarship Program of the Slovak Republic; Grant No. 0108 U004117 and Grant No. GP/F26/0154.

The second author was supported in part by the Grant VEGA-SAV 2/7140/27. 
as a new scalar product on $\mathbb{R}^{n}$, we obtain $(A x, A y)=(x, y)$ and $|A x|=|x|$ for $|x|:=\sqrt{(x, x)}, x \in \mathbb{R}^{n}$, and thus $A$ is unitary with respect to $(\cdot, \cdot)$. We mostly suppose $1 \notin \sigma(A)$, where $\sigma(A)$ is the spectrum of $A$. The norm* on $\mathscr{L}\left(\mathbb{R}^{n}\right)$ generated by $|\cdot|$ is denoted by $\|\cdot\|$.

Remark 1.1. We study (1.1) always in an arbitrary but fixed open bounded subset of $\mathbb{R}^{n}$. So we do not study bifurcations from infinity of (1.1) for $\varepsilon$ small.

Next, for $T>0$ we introduce the Banach space

$$
X:=\left\{x \in C^{0}\left(\mathbb{R}, \mathbb{R}^{n}\right) \mid x(t+T)=A x(t), t \in \mathbb{R}\right\} .
$$

Note $|x(\cdot)|$ is a $T$-periodic function because $|x(t+T)|=|A x(t)|=|x(t)|$ for every $t$. So we take the norm $\|x\|=\max _{t \in[0, T]}|x(t)|=\max _{t \in \mathbb{R}}|x(t)|$ on $X$. It is easy to see that if a function $x$ belongs to the space $X$ then it is a $p T$-periodic function.

Definition 1.1. By a symmetric and periodic solution $x$ of equation (1.1) we understand a solution $x \in X$, where $X$ is defined by (1.3).

The main goal of this paper is to find a unique symmetric and periodic solution (see Sections 2 and 3) for (1.1) and to establish conditions, under which this solution is either stable (see Section 4) or hyperbolic (see Sections 5 and 6). We also present examples to illustrate the theory in Sections 7 and 8. We show that our symmetric averaging method could help when the classical averaging theory fails [18,27].

The results presented here are generalizations of achievements for anti-periodic problems with $A=-\mathbb{1}[1,2]$, and continuations of [7]. Doubly symmetric solutions of reversible systems are studied in [20]. Symmetric properties of periodic solutions of nonlinear nonautonomous ordinary differential equations are also studied in $[23,24]$.

\section{UNIQUENESS FOR GENERAL NONLINEARITIES}

In spite of the fact that we are interested in (1.1), we start with its general form with $\varepsilon=1$, i. e., we first consider the equation

$$
x^{\prime}(t)=f(x(t), t), \quad t \in \mathbb{R} .
$$

Looking for a symmetric and periodic solution $x$ of equation (2.1) is equivalent to solve (2.1) on $t \in[0, T]$ under the boundary condition

$$
x(T)=A x(0) .
$$

The following lemma guarantees the existence and uniqueness of a solution of problem (2.1), (2.2).

Lemma 2.1. Assume that $1 \notin \sigma(A)$ and, moreover, there exists a constant $K>0$ such that

$$
\left|f\left(x_{1}, t\right)-f\left(x_{2}, t\right)\right| \leq K\left|x_{1}-x_{2}\right|
$$

*As usual, the symbol $\mathcal{L}\left(\mathbb{R}^{n}\right)$ stands for the algebra of square matrices of dimension $n$. 
for all $x_{1}, x_{2}$ from $\mathbb{R}^{n}$. If

$$
\left(\left\|(A-\mathbb{1})^{-1}\right\|+1\right) K T<1,
$$

then equation (2.1) has a unique solution $x \in X$.

Proof. Let us first solve the boundary value problem

$$
x^{\prime}=\tilde{h}, \quad x(T)=A x(0),
$$

where $\tilde{h} \in X$. Obviously, $x(t)=x(0)+\int_{0}^{t} \tilde{h}(s) d s$ and $A x(0)=x(T)=x(0)+$ $\int_{0}^{T} \tilde{h}(s) d s$, whence

$$
x(0)=(A-\mathbb{1})^{-1} \int_{0}^{T} \tilde{h}(s) d s .
$$

and, therefore, the solution of (2.5) has the form

$$
x(t)=(A-\mathbb{1})^{-1} \int_{0}^{T} \tilde{h}(s) d s+\int_{0}^{t} \tilde{h}(s) d s, \quad t \in[0, T] .
$$

Let us put

$$
B(x)(t):=(A-\mathbb{1})^{-1} \int_{0}^{T} f(x(s), s) d s+\int_{0}^{t} f(x(s), s) d s, \quad t \in[0, T],
$$

for an arbitrary $x$ from $X$. For any $x_{1}, x_{2} \in X$, using the Lagrange theorem and (2.3), we get

$$
\begin{aligned}
\left\|B\left(x_{1}\right)-B\left(x_{2}\right)\right\|= & \|(A-\mathbb{1})^{-1} \int_{0}^{T}\left(f\left(x_{1}(s), s\right)-f\left(x_{2}(s), s\right)\right) d s \\
& +\int_{0}^{t}\left(f\left(x_{1}(s), s\right)-f\left(x_{2}(s), s\right)\right) d s \| \\
\leq & \left(\left\|(A-\mathbb{1})^{-1}\right\|+1\right) K T\left\|x_{1}(t)-x_{2}(t)\right\| .
\end{aligned}
$$

Taking condition (2.4) into account and applying the Banach fixed point theorem we get that problem (2.1), (2.2) has a unique solution $x \in X$. The proof is complete.

Now we replace Lipschitz condition (2.3) as follows. Let $|\cdot|_{0}$ be a norm on $\mathbb{R}^{n}$. Then for any $x, y \in \mathbb{R}^{n}$ the limit $[3,4]$

$$
N(x, y):=\lim _{\lambda \rightarrow+0} \frac{|x+\lambda y|_{0}-|x|_{0}}{\lambda}
$$


exists and has the following elementary properties:

$$
\left.\begin{array}{rl}
N(c x, c y) & =c N(x, y), \\
\left|N\left(x, y_{1}\right)-N\left(x, y_{1}\right)\right| & \leq\left|y_{1}-y_{2}\right|_{0}, \\
N\left(x, \frac{1}{m} \sum_{k=1}^{m} y_{k}\right) & \leq \frac{1}{m} \sum_{k=1}^{m} N\left(x, y_{k}\right)
\end{array}\right\}
$$

for any $c>0$ and $x, y, y_{1}, y_{2}, \ldots, y_{m}$ from $\mathbb{R}^{n}$. So the function $y \mapsto N(x, y)$ is globally Lipschitz continuous and convex. Next, for any $B \in \mathcal{L}\left(\mathbb{R}^{n}\right)$, there is its Lozinskii logarithmic norm [16]

$$
\mu(B):=\lim _{\lambda \rightarrow+0} \frac{\|\mathbb{1}+\lambda B\|_{0}-1}{\lambda},
$$

where $\|B\|_{0}:=\max _{|x|_{0}=1}|B x|_{0}$. Note that

$$
\sup _{|x|_{0}=1} N(x, B x)=\mu(B) .
$$

Now we are ready to prove the following results motivated by $[6,14,15]$ and $[30$, Theorem 9.6].

Lemma 2.2. Let $f \in C^{1}\left(\mathbb{R}^{n+1}, \mathbb{R}^{n}\right)$. If there is a constant $\alpha>0$ such that

$$
\mu\left(D_{x} f(x, t)\right) \leq-\alpha \quad \forall(x, t) \in \mathbb{R}^{n+1}
$$

then (2.1) possesses a unique symmetric and periodic solution which is globally asymptotically stable.

Proof. Let $x(t)$ be a solution of (2.1) defined on $[0, \delta)$ for some $\delta>0$. Then by (2.6), (2.7) and the Jensen inequality [25], for almost each $t \in[0, \delta)$, we derive

$$
\begin{aligned}
\frac{d}{d t}|x(t)|_{0} & =N\left(x(t), x^{\prime}(t)\right)=N(x(t), f(x(t), t)) \\
& \leq N(x(t), f(x(t), t)-f(0, t))+|f(0, t)|_{0} \\
& \leq N\left(x(t), \int_{0}^{1} D_{x} f(\theta x(t), t) x(t) d \theta\right)+\|f(0, \cdot)\|_{0} \\
& \leq \int_{0}^{1} N\left(x(t), D_{x} f(\theta x(t), t) x(t)\right) d \theta+\|f(0, \cdot)\|_{0} \\
& \leq \int_{0}^{1} \mu\left(D_{x} f(\theta x(t), t)\right) d \theta|x(t)|_{0}+\|f(0, \cdot)\|_{0} \\
& \leq-\alpha|x(t)|_{0}+\|f(0, \cdot)\|_{0},
\end{aligned}
$$


where $\|f(0, \cdot)\|_{0}=\max _{t \in \mathbb{R}} \mid f(0, t)$. Note $|x(t)|_{0}$ is locally absolute continuous. The Gronwall inequality implies

$$
|x(t)|_{0} \leq \mathrm{e}^{-\alpha t}|x(0)|_{0}+\frac{\|f(0, \cdot)\|_{0}}{\alpha}\left(1-\mathrm{e}^{-\alpha t}\right),
$$

so $x(t)$ is defined for any $t \geq 0$ and it terminates in the ball $B\left(0,2\|f(0, \cdot)\|_{0} / \alpha\right)$ centered at 0 with the radius $2\|f(0, \cdot)\|_{0} / \alpha$, i. e., (2.1) is dissipative possessing a $p T$ periodic solution $x_{0}(t)$ inside $B\left(0,2\|f(0, \cdot)\|_{0} / \alpha\right)$ (see [4]).

Next, let $x_{1}(t)$ and $x_{2}(t)$ be two solutions of (2.1) on $\mathbb{R}_{+}$. Then similarly to the consideration above, for almost every $t \in \mathbb{R}_{+}$, we obtain

$$
\begin{array}{r}
\frac{d}{d t}\left|x_{1}(t)-x_{2}(t)\right|_{0}=N\left(x_{1}(t)-x_{2}(t), \frac{d}{d t}\left(x_{1}(t)-x_{2}(t)\right)\right) \\
=N\left(x_{1}(t)-x_{2}(t), f\left(x_{1}(t), t\right)-f\left(x_{2}(t), t\right)\right) \\
=N\left(x_{1}(t)-x_{2}(t), \int_{0}^{1} D_{x} f\left(\theta x_{1}(t)+(1-\theta) x_{2}(t), t\right)\left(x_{1}(t)-x_{2}(t)\right) d \theta\right) \\
\leq \int_{0}^{1} N\left(x_{1}(t)-x_{2}(t), D_{x} f\left(\theta x_{1}(t)+(1-\theta) x_{2}(t), t\right)\left(x_{1}(t)-x_{2}(t)\right)\right) d \theta \\
\leq \int_{0}^{1} \mu\left(D_{x} f\left(\theta x_{1}(t)+(1-\theta) x_{2}(t), t\right)\right) d \theta\left|x_{1}(t)-x_{2}(t)\right|_{0} \\
\leq-\alpha\left|x_{1}(t)-x_{2}(t)\right|_{0},
\end{array}
$$

i. e., the Gronwall inequality again implies

$$
\left|x_{1}(t)-x_{2}(t)\right|_{0} \leq \mathrm{e}^{-\alpha t}\left|x_{1}(0)-x_{2}(0)\right|_{0} .
$$

So (2.9) gives the uniqueness of $x_{0}(t)$ and it is globally asymptotically stable. It is easy to verify that $x(t):=A^{-1} x_{0}(t+T)$ is also a $p T$-periodic solution of (2.1). The uniqueness gives $x(t)=x_{0}(t)$, i. e., $x_{0} \in X$. The proof is finished.

Lemma 2.2 is extended in Corollary 4.1 of Section 4 in the context of averaging theory. The above result can be slightly modified as follows.

Lemma 2.3. Let $f \in C^{1}\left(\mathbb{R}^{n+1}, \mathbb{R}^{n}\right)$. Assume that

$$
\mu\left(D_{x} f(x, t)\right) \leq 0 \quad \forall(x, t) \in \mathbb{R}^{n+1}
$$

and, moreover, that (2.1) possesses a bounded uniformly asymptotically stable solution $x_{0}$ on $\mathbb{R}_{+}$. Then $x_{0}$ is symmetric, periodic, and globally asymptotically stable.

Proof. Let $x(t)$ be a solution of (2.1) defined on $[0, \delta)$ for some $\delta>0$. Then for $\alpha=0,(2.8)$ implies that $|x(t)|_{0} \leq|x(0)|_{0}+\|f(0, \cdot)\|_{0} t$ holds for all $t \geq 0$. So $x(t)$ is defined on $[0, \infty)$.

Next, let $x_{1}(t)$ and $x_{2}(t)$ be two solutions of (2.1) on $\mathbb{R}_{+}$. Then for $\alpha=0,(2.9)$ implies

$$
\left|x_{1}(t)-x_{2}(t)\right|_{0} \leq\left|x_{1}(0)-x_{2}(0)\right|_{0} \quad \forall t \geq 0 .
$$


Furthermore, from the uniform asymptotic stability of $x_{0}(t)$ on $\mathbb{R}_{+}$, there is an $\delta_{0}>0$ such that

$$
\left\{x \in \mathbb{R}^{n} \mid \operatorname{dist}\left(x,\left\{x_{0}(t) \mid t \in \mathbb{R}_{+}\right\}\right)<\delta_{0}\right\} \subset B_{A},
$$

where $B_{A}$ is the domain of attraction of $x_{0}(t)$. Then (2.10) implies that $B\left(z, \delta_{0} / 2\right) \subset$ $B_{A}$ holds for any $z \in B_{A}$. Consequently, $B_{A}=\mathbb{R}^{n}$. Since $x_{0}(t)$ is bounded on $\mathbb{R}_{+}$by assumption, all solutions of (2.1) terminates in a large ball. So (2.1) has a $p T$-periodic solution which now must be $x_{0}(t)$. Then like above it is unique and symmetric. The proof is finished.

We recall the following well-known results:

(1) If $|x|_{0}=\sqrt{\sum_{k=1}^{m} x_{k}^{2}}$ then $\mu(B)=\Lambda\left(\frac{1}{2}\left(B+B^{*}\right)\right)$, where $\Lambda$ means the largest eigenvalue.

(2) If $|x|_{0}=\max _{k=1,2, \ldots, m}\left|x_{k}\right|$ then

$$
\mu(B)=\max _{k=1,2, \ldots, m}\left(b_{k k}+\sum_{j \neq k}\left|b_{k j}\right|\right),
$$

where $b_{k j}, k, j=1,2, \ldots, m$, mean the entries of matrix $B$.

(3) If $|x|_{0}=\sum_{k=1}^{m}\left|x_{k}\right|$ then $\mu(B)=\max _{k=1,2, \ldots, m}\left(b_{k k}+\sum_{j \neq k}\left|b_{j k}\right|\right)$.

Applying Lemmas 2.2 and 2.3, we obtain the following particular results used in the sequel [6].

Lemma 2.4. Let us put

$$
J(x, t):=\frac{1}{2}\left(D_{x} f(x, t)+D_{x} f(x, t)^{*}\right)
$$

and let $\Lambda(x, t)$ be the largest eigenvalue of $J(x, t)$. If there is a constant $\alpha>0$ such that

$$
\Lambda(x, t) \leq-\alpha \quad \forall(x, t) \in \mathbb{R}^{n+1}
$$

then (2.1) possesses a unique symmetric and periodic solution which is globally asymptotically stable.

Lemma 2.5. Assume

$$
\Lambda(x, t) \leq 0 \quad \forall(x, t) \in \mathbb{R}^{n+1}
$$

and (2.1) possesses a bounded, uniformly asymptotically stable solution $x_{0}(t)$ on $\mathbb{R}_{+}$. Then $x_{0}(t)$ is symmetric, periodic and globally asymptotically stable.

Remark 2.1. We do not need to suppose that $1 \notin \sigma(A)$ in Lemmas 2.2-2.5.

Remark 2.2. By reversing the time $t \leftrightarrow-t$ in (2.1), we get similar results like above ensuring that $x_{0}(t)$ is a global repeller, namely, (2.12) and (2.13) are replaced by the inequalities $\lambda(x, t) \geq \alpha$ and $\lambda(x, t) \geq 0$ for all $(x, t) \in \mathbb{R}^{n+1}$, respectively, where $\lambda(x, t)$ is the smallest eigenvalue of $J(x, t)$. 


\section{UNIQUENESS FOR WEAK NONLINEARITIES}

First, we consider the Cauchy problem

$$
\begin{gathered}
x^{\prime}(t)=\varepsilon f(x(t), t), \quad t \in[0, T], \\
x(0)=x .
\end{gathered}
$$

Let us denote by $\varphi_{\varepsilon}(x, t)$ the unique solution of problem (3.1), (3.2). Note that

$$
\varphi_{\varepsilon}(x, 0)=x .
$$

Obviously, the equation

$$
\varphi_{\varepsilon}(x, p T)=x
$$

determines $p T$-periodic solutions of (1.1). For this reason, we consider the diffeomorphism given by the formula

$$
P_{\varepsilon}(x):=\varphi_{\varepsilon}(x, p T) .
$$

Taking (3.4) and (3.5) into account, we get

$$
P_{\varepsilon}(x)=x .
$$

On the other hand, the $T$-periodic and symmetric solutions of (1.1), i. e., the solutions belonging to $X$, are given by the equation

$$
A x=\varphi_{\varepsilon}(x, T) .
$$

In this connection, we introduce the new mapping

$$
g_{\varepsilon}(x):=A^{-1} \varphi_{\varepsilon}(x, T) .
$$

Summarizing the considerations above, we arrive at the following simple lemma.

Lemma 3.1. A fixed point $x$ of $P_{\varepsilon}$, respectively of $g_{\varepsilon}$, determines a $p T$-periodic, respectively a periodic and symmetric, solution of equation (1.1) satisfying (3.2).

The basic relationship between mappings $P_{\varepsilon}$ and $g_{\varepsilon}$ is expressed by the following lemma.

Lemma 3.2. The relation

$$
P_{\varepsilon}(x)=\left[g_{\varepsilon}(x)\right]^{p}
$$

holds, where $P_{\varepsilon}$ and $g_{\varepsilon}$ are defined, respectively, by (3.5) and (3.7).

In (3.8), $\left[g_{\varepsilon}(x)\right]^{p}$ is defined as

$$
\left[g_{\varepsilon}(x)\right]^{p}=\underbrace{\left.g_{\varepsilon}\left(g_{\varepsilon}\left(g_{\varepsilon}\left(g_{\varepsilon} \ldots(x)\right)\right)\right)\right)}_{p}, \quad x \in \mathbb{R}^{n} .
$$


Proof of Lemma 3.2. It is easy to see that

$$
\varphi_{\varepsilon}(x, t+T)=A \varphi_{\varepsilon}\left(A^{-1} \varphi_{\varepsilon}(x, T), t\right),
$$

which implies

$$
\varphi_{\varepsilon}(x, 2 T)=A \varphi_{\varepsilon}\left(A^{-1} \varphi_{\varepsilon}(x, T), T\right)=A A A^{-1} \varphi_{\varepsilon}\left(g_{\varepsilon}(x), T\right) .
$$

Taking into account (3.7), the last relation is equal to

$$
\varphi_{\varepsilon}(x, 2 T)=A^{2}\left[g_{\varepsilon}(x)\right]^{2} .
$$

Let us apply inductive method. Assume that relation

$$
\varphi_{\varepsilon}(x, i T)=A^{i}\left[g_{\varepsilon}(x)\right]^{i}
$$

is true. Now we show that this relation is true also for $i+1$ :

$$
\begin{aligned}
& \varphi_{\varepsilon}(x,(i+1) T)=\varphi_{\varepsilon}(x, i T+T)=A \varphi_{\varepsilon}\left(A^{-1} \varphi_{\varepsilon}(x, T), i T\right) \\
& =A \varphi_{\varepsilon}\left(g_{\varepsilon}(x), i T\right)=A \cdot A^{i} g_{\varepsilon}\left[g_{\varepsilon}(x)\right]^{i}=A^{i+1}\left[g_{\varepsilon}(x)\right]^{i+1} .
\end{aligned}
$$

So, finally we obtain

$$
P_{\varepsilon}(x)=\varphi_{\varepsilon}(x, p T)=A^{p}\left[g_{\varepsilon}(x)\right]^{p}=\left[g_{\varepsilon}(x)\right]^{p} .
$$

The proof is finished.

In the sequel we assume for simplicity that $f$ is $C^{\infty}$-smooth. Asymptotic expansion can be obtained by the methods of averaging and multiple scales. So there exist the Taylor series of $P_{\varepsilon}$ and $g_{\varepsilon}$ with respect to the small parameter $\varepsilon$ :

$$
P_{\varepsilon}(x)=P_{0}(x)+\varepsilon P_{1}(x)+\ldots
$$

and

$$
g_{\varepsilon}(x)=g_{0}(x)+\varepsilon g_{1}(x)+\ldots .
$$

Remark 3.1. It follows from the definition of the mapping $P_{\varepsilon}$ given by formula (3.5) that $P_{0}=\mathbb{1}$.

The next lemma follows from Lemma 3.2.

Lemma 3.3. If $x$ is a solution of equation

$$
g_{\varepsilon}(x)=x,
$$

then it is also a solution of equation (3.6).

Let us solve equation (3.10), which, by (3.7), has the form

$$
H(x, \varepsilon):=A^{-1} \varphi_{\varepsilon}(x, T)-x=0 .
$$

Since

$$
g_{0}(x)=A^{-1} \varphi_{0}(x, T)=A^{-1} x,
$$

we obtain that $H(0,0)=0$ and $D_{x} H(0,0)=A^{-1}-1$. Using the assumption $1 \notin$ $\sigma(A)$ and applying the Implicit Function Theorem, we get the following result. 
Theorem 3.1. If $1 \notin \sigma(A)$ then equation (3.10) has a unique $C^{\infty}$-smooth solution $x_{\varepsilon}=O(\varepsilon)$ for $\varepsilon \neq 0$ small. Note that

$$
P_{\varepsilon}\left(x_{\varepsilon}\right)=x_{\varepsilon} .
$$

Theorem 3.1 implies the following one.

Theorem 3.2. If $1 \notin \sigma(A)$ and $\varepsilon \neq 0$ is small, then equation (1.1) has a unique $p T$-periodic solution $x_{\varepsilon}(t) \in X$ such that $x_{\varepsilon}(0)=x_{\varepsilon}$ and $x_{\varepsilon}(t)=O(\varepsilon)$.

Proof. Lemmas 3.1, 3.2 and Theorem 3.1 immediately give the result.

\section{STABILITY OF PERIODIC AND SYMMETRIC SOLUTIONS}

To study stability of the $T$-periodic and symmetric solution $x_{\varepsilon}(\cdot) \in X$ of equation (1.1), we consider the linearization

$$
M(\varepsilon):=D_{x} g_{\varepsilon}\left(x_{\varepsilon}\right)
$$

of mapping (3.7) at the fixed point $x_{\varepsilon}=O(\varepsilon)$. Note that, by (3.8), we have

$$
\left[D_{x} g_{\varepsilon}\left(x_{\varepsilon}\right)\right]^{p}=D_{x} P_{\varepsilon}\left(x_{\varepsilon}\right),
$$

which, by the Dunford spectral mapping theorem [11], yields

$$
\sigma\left(D_{x} P_{\varepsilon}\left(x_{\varepsilon}\right)\right)=\left[\sigma\left(D_{x} g_{\varepsilon}\left(x_{\varepsilon}\right)\right)\right]^{p} .
$$

Taking (3.7) into account, we get

$$
D_{x} g_{\varepsilon}(x)=A^{-1} D_{x} \varphi_{\varepsilon}(x, T) .
$$

So, using (3.11), we obtain

$$
D_{x} g_{0}(x)=A^{-1} D_{x} \varphi_{0}(x, T)=A^{-1} .
$$

From (4.1), (4.3) we have that

$$
M(0)=A^{-1},
$$

but $A$ is a unitary linear mapping with all eigenvalues on the unit circle.

Let us consider the decomposition of the mapping $M(\varepsilon)$ into the Taylor series on a small parameter $\varepsilon$ using (4.4):

$$
M(\varepsilon)=A^{-1}+\varepsilon M_{1}+\ldots .
$$

By definition,

$$
M_{1}=\frac{d}{d \varepsilon} M(0),
$$

it is easy to see, taking into account (4.1), $x_{0}=0$ and $D_{x}^{2} g_{0}(0)=0$, that

$$
\begin{aligned}
M_{1}=\left.\frac{d}{d \varepsilon}\left[D_{x} g_{\varepsilon}\left(x_{\varepsilon}\right)\right]\right|_{\varepsilon=0}=D_{x}^{2} g_{0}(0) \frac{d}{d \varepsilon} x_{0}+\frac{d}{d \varepsilon} & D_{x} g_{0}\left(x_{0}\right) \\
& =A^{-1} \frac{d}{d \varepsilon} D_{x} \varphi_{0}(0, T) .
\end{aligned}
$$


Let us consider problem (3.1), (3.2). By (3.3), we get that

$$
\begin{gathered}
\dot{\varphi}_{\varepsilon}(x, t)=\varepsilon f\left(\varphi_{\varepsilon}(x, t), t\right), \\
\varphi_{\varepsilon}(x, 0)=x .
\end{gathered}
$$

We differentiate (4.7), (4.8) with respect to $x$ and get

$$
\begin{gathered}
\frac{d}{d t}\left(D_{x} \varphi_{\varepsilon}(x, t)\right)={ }_{\varepsilon} D_{x} f\left(\varphi_{\varepsilon}(x, t), t\right) D_{x} \varphi_{\varepsilon}(x, t), \\
D_{x} \varphi_{\varepsilon}(x, 0)=\mathbb{1} .
\end{gathered}
$$

Now differentiating (4.9), (4.10) with respect to $\varepsilon$ and putting $\varepsilon=0$, we get

$$
\begin{gathered}
\frac{d}{d t}\left(\frac{d}{d \varepsilon} D_{x} \varphi_{0}(x, t)\right)=D_{x} f(x, t) D_{x} \varphi_{0}(x, t), \\
\frac{d}{d \varepsilon} D_{x} \varphi_{0}(x, 0)=0 .
\end{gathered}
$$

Thus,

$$
\frac{d}{d \varepsilon} D_{x} \varphi_{0}(x, T)=\int_{0}^{T} D_{x} f(x, s) d s .
$$

Using (4.6) and (4.11) we arrive at the equality

$$
M_{1}=A^{-1} \int_{0}^{T} D_{x} f(0, s) d s
$$

Now we return to the Taylor series (4.5). Considering previous calculations and using that $x_{\varepsilon}=O(\varepsilon)$ from Theorem 3.1, we derive

$$
\begin{aligned}
M(\varepsilon)=A^{-1}+\varepsilon A^{-1} & \int_{0}^{T} D_{x} f(0, s) d s+\cdots \\
& =A^{-1}\left(\mathbb{1}+\varepsilon \int_{0}^{T} D_{x} f(0, s) d s+\cdots\right)=A^{-1} G(\varepsilon),
\end{aligned}
$$

where $G(\varepsilon)=A M(\varepsilon)=\mathbb{1}+\varepsilon \int_{0}^{T} D_{x} f(0, s) d s+\cdots$. Since $A^{-1}$ is unitary, we get that $M(\varepsilon)$ is stable if $G(\varepsilon)$ is stable. Let us now prove a generalization of a classical result $[5,6,22]$.

Theorem 4.1. Let $M(\varepsilon) \in \mathcal{L}\left(\mathbb{R}^{n}\right)$ be given by (4.12). If

$$
\operatorname{Re}\left\{\sigma\left(\int_{0}^{T} D_{x} f(0, s) d s\right)\right\} \subset(-\infty, 0),
$$

then there exists an $\varepsilon_{0}>0$ such that $r(M(\varepsilon))<1$ for any $0<\varepsilon \leq \varepsilon_{0}$, where $r(M(\varepsilon))$ is the spectral radius of $M(\varepsilon)$.

In order to prove Theorem 4.1, we recall the following well-known results $[10,11$, 17]. 
Theorem 4.2. Let $K \in \mathcal{L}\left(\mathbb{R}^{n}\right)$. Then $r(K)<1$ if and only if there exists a norm on $\mathbb{R}^{n}$ such that $\|K\|<1$.

Theorem 4.3. Assume that (4.13) is fulfilled then there exists a scalar product $\langle\cdot, \cdot\rangle_{1}$ and constant $\alpha, \alpha>0$ such that

$$
\left\langle\left(\int_{0}^{T} D_{x} f(0, s) d s\right) x, x\right\rangle_{1} \leq-\alpha\|x\|_{1}^{2} \quad \text { for any } x \in \mathbb{R}^{n},
$$

where $\|x\|_{1}=\sqrt{\langle x, x\rangle_{1}}, x \in \mathbb{R}^{n}$.

Proof of Theorem 4.1. Consider the expression $\|G(\varepsilon) x\|_{1}^{2}=\langle G(\varepsilon) x, G(\varepsilon) x\rangle_{1}$. Differentiating $\|G(\varepsilon)\|_{1}^{2}$ with respect to $\varepsilon$, we get

$$
\frac{d}{d \varepsilon}\|G(\varepsilon) x\|_{1}^{2}=2\left\langle G^{\prime}(\varepsilon) x, G(\varepsilon) x\right\rangle_{1} .
$$

Using the Lagrange theorem, we obtain

$$
\begin{aligned}
\|G(\varepsilon) x\|_{1}^{2} & =\|G(0) x\|_{1}^{2}+\frac{d}{d \varepsilon}\|G(\theta) x\|_{1}^{2} \varepsilon \\
& =\|x\|_{1}^{2}+2\left\langle G^{\prime}(\theta) x, G(\theta) x\right\rangle_{1} \varepsilon, \quad 0 \leq \theta \leq \varepsilon .
\end{aligned}
$$

By virtue of (4.14), we arrive at the equality

$$
\left\langle G^{\prime}(0) x, G(0) x\right\rangle_{1}=\left\langle\left(\int_{0}^{T} D_{x} f(0, s) d s\right) x, x\right\rangle_{1} \leq-\alpha\|x\|_{1}^{2} .
$$

Thus,

$$
2\left\langle G^{\prime}(\theta) x, G(\theta) x\right\rangle_{1} \leq-\alpha\|x\|_{1}^{2}
$$

for $0<\varepsilon \leq \varepsilon_{0}$ with $\varepsilon_{0}$ small enough. Returning to (4.15), we obtain

$$
\|G(\varepsilon) x\|_{1}^{2} \leq\|x\|_{1}^{2}-\alpha\|x\|_{1}^{2} \varepsilon=(1-\alpha \varepsilon)\|x\|_{1}^{2} .
$$

Supposing that $\varepsilon_{0} \alpha<1$, we get

$$
\|G(\varepsilon) x\|_{1} \leq \sqrt{1-\alpha \varepsilon}\|x\|_{1} \quad \forall x \in \mathbb{R}^{n} .
$$

Consequently, we have that $\|G(\varepsilon)\|_{1} \leq \sqrt{1-\alpha \varepsilon}<1$. Then Theorem 4.2 implies that $r(G(\varepsilon))<1$. Finally, since $A$ is unitary with respect to the norm $|\cdot|$, we have [11]

$$
r(M(\varepsilon))=\lim _{k \rightarrow \infty} \sqrt[k]{\left\|M(\varepsilon)^{k}\right\|}=\lim _{k \rightarrow \infty} \sqrt[k]{\left\|G(\varepsilon)^{k}\right\|}=r(G(\varepsilon))<1,
$$

which completes the proof of Theorem 4.1.

Summarizing, we obtain the following result.

Theorem 4.4. Suppose $1 \notin \sigma(A)$. Then for any $\varepsilon>0$ small, the unique symmetric and T-periodic solution $x(t)=O(\varepsilon)$ of equation (1.1) is asymptotically stable if condition (4.13) is fulfilled. 
Proof. We know from Theorem 4.1 that $r\left(D_{x} g_{\varepsilon}\left(x_{\varepsilon}\right)\right)<1$. Then, using (4.2), we have

$$
r\left(D_{x} P_{\varepsilon}\left(x_{\varepsilon}\right)\right)=r\left(D_{x} g_{\varepsilon}\left(x_{\varepsilon}\right)\right)^{p}<1 .
$$

The proof is complete.

Finally, we generalize Lemma 2.2 as follows to get a practical global stability of symmetric and periodic solutions of (1.1) [12]. By using the change of variables [8]

$$
x \longleftrightarrow x+\varepsilon\left(\int_{0}^{t} f(x, s) d s-\bar{f}(x) t\right),
$$

equation (1.1) is transformed to the form

$$
x^{\prime}(t)=\varepsilon \bar{f}(x(t))+\varepsilon^{2} g(x(t), t, \varepsilon)
$$

with a $C^{\infty}$-smooth and $p T$-periodic $g$. Here $\bar{f}(x):=\frac{1}{p T} \int_{0}^{p T} f(x, s) d s$. Note that $A \bar{f}(x)=\bar{f}(A x)$. Therefore, $A \bar{f}(0)=\bar{f}(0)$ and, thus, $\bar{f}(0)=0$ because $1 \notin \sigma(A)$. Suppose the existence of a norm $|\cdot|_{0}$ on $\mathbb{R}^{n}$ and a constant $\alpha>0$ such that

$$
\mu(D \bar{f}(x))<-\alpha \quad \text { for any } x \in \mathbb{R}^{n},
$$

where $\mu$ is the corresponding Lozinskii logarithmic norm. Then from the proof of Lemma 2.2 we see that equilibrium $z=0$ is a global attractor for the system

$$
\dot{z}=\varepsilon \bar{f}(z) \text {. }
$$

Now we are ready to prove the following results [28].

Theorem 4.5. Suppose that (4.17) holds for a norm $|\cdot|_{0}$ on $\mathbb{R}^{n}$ and a constant $\alpha>0$ with the corresponding Lozinskii logarithmic norm $\mu$. Take $R_{0}>R_{1}>0$ and put

$$
K_{0}:=\max _{|x|_{0} \leq R_{0},|\varepsilon| \leq 1, t \in \mathbb{R}}|g(x, t, \varepsilon)|_{0},
$$

and

$$
K_{1}:=\max _{|x|_{0} \leq R_{0},|\varepsilon| \leq 1, t \in \mathbb{R}}\left\|D_{x} g(x, t, \varepsilon)\right\|_{0}
$$

If

$$
0<\varepsilon<\min \left\{1, \frac{R_{0}-R_{1}}{K_{0}} \alpha, \frac{R_{0} \alpha}{3 K_{0}}, \frac{K_{1}}{\alpha}\right\}
$$

then any solution of (4.16) starting from the ball $B\left(0, R_{1}\right)$ remains in $B\left(0, R_{0}\right)$ and tends asymptotically to a unique $p T$-periodic solution of (4.16).

Moreover, if $x(\cdot)$ and $z(\cdot)$ are solutions of (4.16) and (4.18) on $\mathbb{R}_{+}$such that $x(0)=z(0)$ and $|x(0)| \leq R_{1}$, then

$$
|x(t)-z(t)|_{0} \leq \frac{K_{0}}{\alpha} \varepsilon
$$

for all $t \in \mathbb{R}_{+}$. 
Proof. We take $\varepsilon$ satisfying (4.19). Let $x(t)$ be a solution of (4.16) such that $|x(0)| \leq R_{1}$ and $|x(t)| \leq R_{0}$ for all $t \in[0, \delta)$. Then from the proof of Lemma 2.2, we obtain

$$
|x(t)|_{0} \leq \mathrm{e}^{-\alpha \varepsilon t} R_{1}+\frac{\varepsilon K_{0}}{\alpha} \leq R_{1}+\frac{\varepsilon K_{0}}{\alpha} \leq R_{0}
$$

for any $t \in[0, \delta)$. Thus, in fact, $\delta=+\infty$. Since

$$
\frac{\varepsilon K_{0}}{\alpha} \leq \frac{R_{0}}{3}<\frac{R_{0}}{2}
$$

all solutions starting from $B\left(0, R_{1}\right)$ terminate in $B\left(0, R_{0} / 2\right)$ as $t \rightarrow \infty$. Next, again from the proof of Lemma 2.2, we obtain

$$
\left|x_{1}(t)-x_{2}(t)\right|_{0} \leq \mathrm{e}^{-\varepsilon\left(\alpha-\varepsilon K_{1}\right) t}\left|x_{1}(t)-x_{2}(t)\right|_{0} \leq 2 \mathrm{e}^{-\varepsilon\left(\alpha-\varepsilon K_{1}\right) t} R_{1} \quad \forall t \geq 0
$$

for any two solutions $x_{1}(t)$ and $x_{2}(t)$ of (4.16) starting in $B\left(0, R_{1}\right)$. Consequently, (4.16) has a unique $p T$-periodic solution in $B\left(0, R_{1}\right)$ and it is attracting all solutions starting in this ball. Finally, like above we see that any solution of (4.18) starting in ball $B\left(0, R_{1}\right)$ remains in $B\left(0, R_{1}\right)$ and it asymptotically tends to an equilibrium $z=0$. Estimate (4.20) is derived similarly for any solutions $x(\cdot)$ and $z(\cdot)$ of (4.16) and (4.18) on $\mathbb{R}_{+}$with $x(0)=z(0)$ and $|x(0)| \leq R_{1}$. The proof is complete.

We get immediately from Theorem 4.5 the following result.

Corollary 4.1. Assume that (4.17) holds for a norm $|\cdot|_{0}$ on $\mathbb{R}^{n}$ and a constant $\alpha>0$ with the corresponding Lozinskii logarithmic norm $\mu$. Then for any bounded subset $S \subset \mathbb{R}^{n}$ there are constants $\widetilde{K}>0$ and $\widetilde{\varepsilon}>0$ such that for any $0<\varepsilon<\widetilde{\varepsilon}$, if $x(t)$ and $z(t)$ are solutions of (1.1) and (4.18) on $\mathbb{R}_{+}$with $x(0)=z(0) \in \mathcal{S}$, then

$$
|x(t)-z(t)|_{0} \leq \widetilde{K}_{0} \varepsilon
$$

for all $t \in \mathbb{R}_{+}$. Moreover, $x(t)$ asymptotically tends to a unique periodic and symmetric solution of (1.1).

Remark 4.1. Results similar to the estimates (4.20) and (4.21) above are obtained in the paper [27].

\section{AVERAGING THEORY}

In this section we survey classical results $[5,9,18,21,22,26,27]$ on the local uniqueness and stability of $p T$-periodic solutions of equation (1.1). We already know that $p T$-periodic solutions are described by mapping (3.5). So, now we recall some known results for this mapping. Taking into account (3.9), we have

$$
P_{\varepsilon}(x)=\varphi_{0}(x, p T)+\varepsilon \varphi_{1}(x, p T)+\cdots
$$

From (3.6) we have

$$
P_{0}(x)+\varepsilon P_{1}(x)+\cdots=x,
$$


which, in view of the relation $P_{0}(x)=\varphi_{0}(x, p T)=x$, becomes $\varepsilon P_{1}(x)+\cdots=0$. It is easy to see that $P_{1}(x)=\left.\frac{d}{d \varepsilon} P_{\varepsilon}(x)\right|_{\varepsilon=0}$, and using (5.1) we get

$$
P_{1}(x)=\frac{d}{d \varepsilon} \varphi_{0}(x, p T) .
$$

Similar as it is done in Section 4 we solve the problem (4.7), (4.8) and considering (5.2), one can get that

$$
P_{1}(x)=\frac{d}{d \varepsilon} D \varphi_{0}(0, p T)=\int_{0}^{p T} f(x, s) d s
$$

and

$$
D_{x} P_{1}(x)=\int_{0}^{p T} D_{x} f(x, s) d s .
$$

Now repeating the procedure of the previous sections, we are ready to state the following classical result.

Theorem 5.1. If there exists $x_{0} \in \mathbb{R}^{n}$ such that

$$
\int_{0}^{p T} f\left(x_{0}, s\right) d s=0
$$

and

$$
\operatorname{det} \int_{0}^{p T} D_{x} f\left(x_{0}, s\right) d s \neq 0
$$

then, for any $\varepsilon \neq 0$ small, equation (1.1) has a unique $p T$-periodic solution $x(t)=$ $x_{0}+O(\varepsilon)$. Moreover, for $\varepsilon>0$ small, the following statements are true:

(1) If $\operatorname{Re}\left\{\sigma\left(\int_{0}^{p T} D_{x} f\left(x_{0}, s\right) d s\right)\right\} \subset(-\infty, 0)$ then $x(t)$ is asymptotically stable.

(2) If $\operatorname{Re}\left\{\sigma\left(\int_{0}^{p T} D_{x} f\left(x_{0}, s\right) d s\right)\right\} \cap(0, \infty) \neq \varnothing$ then $x(t)$ is unstable.

(3) If $\operatorname{Re}\left\{\sigma\left(\int_{0}^{p T} D_{x} f\left(x_{0}, s\right) d s\right)\right\} \subset(0, \infty)$ then $x(t)$ is a repeller.

(4) If $\operatorname{Re}\left\{\sigma\left(\int_{0}^{p T} D_{x} f\left(x_{0}, s\right) d s\right)\right\} \cap\{0\}=\varnothing$ then $x(t)$ is hyperbolic with the same hyperbolicity type as $\int_{0}^{p T} D_{x} f\left(x_{0}, s\right) d s$.

Remark 5.1. By (1.2), (5.3), and (5.4), we have

$$
A P_{1}(x)=P_{1}(A x), \quad A D_{x} P_{1}(x)=D_{x} P_{1}(A x) A \quad \forall x \in \mathbb{R}^{n} .
$$

So if $P_{1}\left(x_{0}\right)=0$ then $P_{1}\left(x_{j}\right)=0$ and $\sigma\left(D_{x} P_{1}\left(x_{j}\right)\right)=\sigma\left(D_{x} P_{1}\left(x_{0}\right)\right)$ for $x_{j}:=$ $A^{j} x_{0}$ and $j=0,1, \ldots, p-1$. Assuming $x_{0} \neq 0$ from $1 \notin \sigma(A)$, there is the maximal $p_{0}>1, p_{0} \mid p$ such that $x_{j}$ are different for $j=0,1, \ldots, p_{0}-1$. Then if

$$
\operatorname{det} D_{x} P_{1}\left(x_{0}\right) \neq 0 \text {, }
$$


then, by Theorem 5.1, we have $p_{0}$ distinct $p T$-periodic solutions $x_{j}(t)=x_{j}+O(\varepsilon)$ for $j=0,1, \ldots, p_{0}-1$. By (1.2), the function $\tilde{x}_{j}(t):=A^{j} x_{0}(t-j T)=A^{j} x_{0}+$ $O(\varepsilon)=x_{j}+O(\varepsilon)$ is also a solution of (1.1). The uniqueness from Theorem 5.1 yields $\tilde{x}_{j}(t)=x_{j}(t)$. Consequently, $x_{j}(t), j=0,1, \ldots, p_{0}-1$ are distinct solutions of (1.1), but geometrically they are $A$-symmetric to one other with same local asymptotic properties.

\section{6. $k$-HYPERBOLICITY}

To study hyperbolicity of periodic solutions of equation (1.1) we need the following results from [22].

Definition 6.1. A continuous matrix function $L_{\varepsilon}: \mathbb{R}^{n} \rightarrow \mathbb{R}^{n}$ of $\varepsilon \geq 0$ is $k$-hyperbolic if, for every matrix function $N_{\varepsilon}$ defined for $\varepsilon \geq 0$ satisfying $N_{\varepsilon}=o\left(\varepsilon^{k}\right)$, there exists an interval $0<\varepsilon<\varepsilon_{1}$ in which $L_{\varepsilon}+N_{\varepsilon}$ is hyperbolic of the same type (i. e., with the same number of eigenvalues on each side of the unit circle).

Definition 6.2. A continuous matrix function $L_{\varepsilon}: \mathbb{R}^{n} \rightarrow \mathbb{R}^{n}$ of $\varepsilon \geq 0$ is strongly $k$ hyperbolic if there exists a continuous real matrix $C_{\varepsilon}$ defined in an interval $0 \leq \varepsilon<\varepsilon_{0}$ such that $C_{\varepsilon}$ is regular (even for $\varepsilon=0$ ) and such that

$$
C^{-1} L_{\varepsilon} C_{\varepsilon}=\left(\begin{array}{cc}
A_{\varepsilon} & 0 \\
0 & B_{\varepsilon}
\end{array}\right)
$$

for $0<\varepsilon<\varepsilon_{0}$, where $A_{\varepsilon}$ and $B_{\varepsilon}$ are $r \times r$ and $s \times s$ blocks, respectively, and $\left\|A_{\varepsilon}\right\|<$ $1-c \varepsilon^{k},\left\|B_{\varepsilon}^{-1}\right\|<1-c \varepsilon^{k}$ for some $c>0$.

Now repeating proofs of Theorems 2.2 and 2.4 of [22], we arrive at the following generalizations.

Theorem 6.1. Strong k-hyperbolicity implies $k$-hyperbolicity.

Theorem 6.2. If $L_{\varepsilon}=L_{0}+\varepsilon L_{1}+\cdots+\varepsilon^{k} L_{k}$, if the eigenvalues of $L_{0}$ are distinct numbers on the unit circle, and if the eigenvalues $\lambda_{i}(\varepsilon)$ of $L_{\varepsilon}$ suitably numbered satisfy $\left|\lambda_{i}(\varepsilon)\right|<1-c \varepsilon^{k}$ for $i=1, \ldots r,\left|\lambda_{i}(\varepsilon)\right|>1-c \varepsilon^{k}$ for $i=r+1, \ldots, n$, for some constant $c>0$ and $\varepsilon>0$ small, then $L_{\varepsilon}$ is strongly $k$-hyperbolic.

Remark 6.1. A difference between our Theorem 6.2 and [22, Theorem 2.2] is that it is supposed $L_{\varepsilon}=\mathbb{1}+\varepsilon L_{1}+\cdots+\varepsilon^{k} L_{k}$ and the eigenvalues of $L_{1}$ are distinct in [22, Theorem 2.2].

Now we can improve Theorem 4.4 as follows.

Theorem 6.3. Suppose $1 \notin \sigma(A)$ and all eigenvalues of $A$ are distinct complex numbers on the unit circle. Let $x_{\varepsilon}=O(\varepsilon)$ be the unique solution of (3.10). If

$$
D_{x} g_{\varepsilon}\left(x_{\varepsilon}\right)=G_{\varepsilon, k}+o\left(\varepsilon^{k}\right):=A^{-1}+\varepsilon G_{1}+\cdots+\varepsilon^{k} G_{k}+o\left(\varepsilon^{k}\right)
$$


with a $k$-hyperbolic matrix function $G_{\varepsilon, k}$ then $D_{x} g_{\varepsilon}\left(x_{\varepsilon}\right)$ is hyperbolic of the same type as $G_{\varepsilon, k}$. In particular, $G_{\varepsilon, k}$ is $k$-hyperbolic if it fulfills assumptions of Theorem 6.2.

According to (4.2), from Theorem 6.3, we obtain the following consequence.

Theorem 6.4. Suppose $1 \notin \sigma(A)$ and all eigenvalues of $A$ are distinct complex numbers on the unit circle. If the eigenvalues $\lambda_{i}(\varepsilon)$ of $A^{-1}+\varepsilon G_{1}+\cdots+\varepsilon^{k} G_{k}$ suitably numbered satisfy $\left|\lambda_{i}(\varepsilon)\right|<1-c \varepsilon^{k}$ for $i=1, \ldots r,\left|\lambda_{i}(\varepsilon)\right|>1-c \varepsilon^{k}$ for $i=r+1, \ldots, n$, for some constant $c>0$ and $\varepsilon>0$ small.

Then the unique symmetric and T-periodic solution of (1.1) is hyperbolic for any $\varepsilon>0$ small.

\section{APPLICATIONS TO WEAKLY NONLINEAR PROBLEMS}

In this section, we present two concrete weakly nonlinear ordinary differential equations to illustrate our theory.

\subsection{A scalar problem}

Let us consider a scalar differential equation

$$
x^{\prime}=\varepsilon\left(x-x^{3}+\sin t\right), \quad x \in \mathbb{R},
$$

with $T=\pi, p=2$ and $f(x, t)=x-x^{3}+\sin t, A x=-x$. Indeed, we verify condition (1.2):

$$
\begin{aligned}
& A f(x, t)=-f(x, t)=-\left(x-x^{3}+\sin t\right)= \\
& \quad=(-x)-\left(-x^{3}\right)+\sin (t+\pi)=f(-x, t+\pi)=f(A x, t+\pi) .
\end{aligned}
$$

Let us establish the stability of the $p T$-periodic solution of the symmetric problem (1.1), (2.2). Using (5.3) we get

$$
P_{1}(x)=\int_{0}^{2 \pi}\left(x-x^{3}+\sin t\right) d t=2 \pi\left(x-x^{3}\right) .
$$

Solving the equation $P_{1}(x)=0$ we find that

$$
x=0, \quad x=-1, \quad x=1 .
$$

It is easy to see, using (5.4), that

$$
\begin{gathered}
P_{1}^{\prime}(x)=2 \pi\left(1-3 x^{2}\right), \\
P_{1}^{\prime}(-1)<0, \quad P_{1}^{\prime}(0)>0, \quad P_{1}^{\prime}(1)<0 .
\end{gathered}
$$

From Theorem 5.1 we know that $x_{0}(t)=O(\varepsilon), x_{ \pm}(t)= \pm 1+O(\varepsilon)$ are the only $2 \pi$-periodic solutions of the problem (7.1) for $\varepsilon>0$ small, while $x_{0}(t)$ is unstable (a repeller) and $x_{ \pm}(t)$ are asymptotically stable. Note by Remark 5.1, curves $x_{ \pm}(t)$ 
are symmetric with respect to the origin to each others. Furthermore, from Theorem 3.2 we know that $x_{0}(t)$ is the only $\pi$-antiperiodic solution of (7.1). Now using Theorem 4.4 together with a calculation

$$
\int_{0}^{\pi} f^{\prime}(0, s) d s=\pi>0
$$

we reverify its instability.

\subsection{A planar problem}

Let us consider a planar differential system

$$
\begin{aligned}
& x_{1}^{\prime}=\varepsilon\left(f_{1}\left(x_{1}, x_{2}\right)+h_{1}(t)\right), \\
& x_{2}^{\prime}=\varepsilon\left(f_{2}\left(x_{1}, x_{2}\right)+h_{2}(t)\right)
\end{aligned}
$$

with smooth functions $f_{1,2}, h_{1,2}, T=\pi / 2, p=4$, and with

$$
A=\left(\begin{array}{cc}
0 & -1 \\
1 & 0
\end{array}\right) \text {. }
$$

Then symmetry condition (1.2) implies

$$
f_{1}\left(x_{1}, x_{2}\right)=f_{2}\left(-x_{2}, x_{1}\right), \quad f_{2}\left(x_{1}, x_{2}\right)=-f_{1}\left(-x_{2}, x_{1}\right),
$$

and

$$
h_{1}\left(t+\frac{\pi}{2}\right)=-h_{2}(t), \quad h_{2}\left(t+\frac{\pi}{2}\right)=h_{1}(t) .
$$

Note that (7.5) gives $h_{1,2}(t+\pi)=-h_{1,2}(t)$ and, thus,

$$
\int_{0}^{2 \pi} h_{1,2}(t) d t=0
$$

Consequently, (5.3) now has the form

$$
P_{1}(x)=2 \pi\left(f_{1}\left(x_{1}, x_{2}\right), f_{2}\left(x_{1}, x_{2}\right)\right),
$$

where $x=\left(x_{1}, x_{2}\right)$. Symmetry conditions (7.4) are satisfied, e. g., for the polynomials

$$
\begin{aligned}
f_{1}\left(x_{1}, x_{2}\right)= & a_{0} x_{1}+b_{0} x_{2}+\sum_{\substack{j, k=0, j+k \geq 3 \\
2 \mid j+k+1}}^{m}\left(a_{j k} x_{1}^{j} x_{2}^{k}+b_{k j} x_{1}^{k} x_{2}^{j}\right), \\
f_{2}\left(x_{1}, x_{2}\right)= & -b_{0} x_{1}+a_{0} x_{2} \\
& -\sum_{\substack{j, k=0, j+k \geq 3 \\
2 \mid j+k+1}}^{m}\left((-1)^{k} b_{k j} x_{1}^{j} x_{2}^{k}+(-1)^{j} a_{j k} x_{1}^{k} x_{2}^{j}\right) .
\end{aligned}
$$


Hence $D_{x} P_{1}(0)=\left(\begin{array}{ll}a_{0} & b_{0} \\ b_{0} & a_{0}\end{array}\right)$. Then from Theorem 5.1 we know that $x_{0}(t)=O(\varepsilon)$ is the only symmetric and $\pi / 2$-periodic solution of (7.2) which is in addition asymptotically stable (a repeller) if $a_{0}<0\left(a_{0}>0\right)$, respectively, for $\varepsilon>0$ small. We need to compute higher-order terms for $a_{0}=0$, but it is not carried out here. Since in general polynomials (7.7) are difficult to handle, we consider the following particular cases.

\subsubsection{Degenerate cases}

If $a_{0}=b_{0}=0$ then $D_{x} P_{1}(0)=0$ and so Theorem 5.1 is not applicable. But Theorem 3.2 still guarantees the existence and uniqueness of $x_{0}(t)$. This is one of advantages of our results. For instance, consider

$$
\begin{aligned}
& x_{1}^{\prime}=\varepsilon\left(a_{j} x_{1}^{j}+b_{j} x_{2}^{j}+h_{1}(t)\right), \\
& x_{2}^{\prime}=\varepsilon\left(-b_{j} x_{1}^{j}+a_{j} x_{2}^{j}+h_{2}(t)\right),
\end{aligned}
$$

where $a_{j}, b_{j} \in \mathbb{R} \backslash\{0\}, j \in \mathbb{N}, j \geq 3$ is odd and $h_{1,2} \neq 0$ satisfy (7.5). The linearization of (7.8) along $x_{0}(t)=\left(x_{1,0}(t), x_{2,0}(t)\right)$ has the form

$$
\begin{aligned}
& x_{1}^{\prime}=\varepsilon\left(j a_{j} x_{1,0}(t)^{j-1} x_{1}+j b_{j} x_{2,0}(t)^{j-1} x_{2}\right), \\
& x_{2}^{\prime}=\varepsilon\left(-j b_{j} x_{1,0}(t)^{j-1} x_{1}+j a_{j} x_{2,0}(t)^{j-1} x_{2}\right) .
\end{aligned}
$$

Let $X_{\varepsilon}(t)$ be the fundamental matrix solution of (7.9). Then in notations of Sections 4 and 5, we have $D_{x} g_{\varepsilon}\left(x_{\varepsilon}\right)=A^{-1} X_{\varepsilon}(\pi / 2)$ and $D_{x} P_{\varepsilon}\left(x_{\varepsilon}\right)=X_{\varepsilon}(2 \pi)$ for $x_{\varepsilon}=x_{0}(0)$. Since

$$
\sigma\left(A^{-1} X_{0}(\pi / 2)\right)=\left\{\mathrm{e}^{ \pm \pi l / 2}\right\}
$$

it follows that $\sigma\left(A^{-1} X_{\varepsilon}(\pi / 2)\right)=\left\{\lambda_{\varepsilon}, \bar{\lambda}_{\varepsilon}\right\}$ is close to $\left\{\mathrm{e}^{ \pm \pi l / 2}\right\}$. Let $\sigma\left(X_{\varepsilon}(2 \pi)\right)=$ $\left\{\lambda_{\varepsilon, 1}, \lambda_{\varepsilon, 2}\right\}$. Then, due to (4.2), we have $\lambda_{\varepsilon, 1}=\lambda_{\varepsilon}^{4}$ and $\lambda_{\varepsilon, 2}=\left(\bar{\lambda}_{\varepsilon}\right)^{4}=\bar{\lambda}_{\varepsilon}^{4}=\bar{\lambda}_{\varepsilon, 1}$. On the other hand, by the Liouville theorem [11], we know that

$$
\lambda_{\varepsilon, 1} \lambda_{\varepsilon, 2}=\operatorname{det} X_{\varepsilon}(2 \pi)=\mathrm{e}_{0}^{2 \pi} j a_{j}\left(x_{1,0}(t)^{j-1}+x_{2,0}(t)^{j-1}\right) d t .
$$

Therefore, since $\int_{0}^{2 \pi}\left(x_{1,0}(t)^{j-1}+x_{2,0}(t)^{j-1}\right) d t>0$, we see that $\left|\lambda_{\varepsilon, 1}\right|=\left|\lambda_{\varepsilon, 2}\right|<$ 1 for $a_{j}<0$ and $\left|\lambda_{\varepsilon, 1}\right|=\left|\lambda_{\varepsilon, 2}\right|>1$ for $a_{j}>0$. Furthermore, the relation $P_{1}(x)=0$ has the form

$$
a_{j} x_{1}^{j}+b_{j} x_{2}^{j}=0, \quad-b_{j} x_{1}^{j}+a_{j} x_{2}^{j}=0,
$$

which gives $\left(x_{1} x_{2}\right)^{j}\left(a_{j}^{2}+b_{j}^{2}\right)=0$. Thus, (7.10) has only zero solution $x_{1}=x_{2}=0$. Summarizing, we have the following result. 
Theorem 7.1. Weakly nonlinear system (7.8) has the only $2 \pi$-periodic solution $x_{0}(t)=O(\varepsilon)$ for $\varepsilon>0$ small, which is moreover $-\pi / 2$-symmetric, i.e., has the property

$$
x_{0}(t+\pi / 2)=\left(\begin{array}{cc}
0 & -1 \\
1 & 0
\end{array}\right) x_{0}(t) \quad \forall t \in \mathbb{R} .
$$

If $a_{j}<0$ then $x_{0}(t)$ is globally asymptotically stable, and if $a_{j}>0$ then $x_{0}(t)$ is a global repeller.

Proof. We concentrate on the case $a_{j}<0$, since the case $a_{j}>0$ can be handled similarly. We already know that (7.8) possesses a solution $x_{0}(t)$ which is asymptotically stable. Now we apply Lemma 2.5. By (2.11), we have

$$
J(x, t)=j a_{j} \varepsilon\left(\begin{array}{cc}
x_{1}^{j-1} & 0 \\
0 & x_{2}^{j-1}
\end{array}\right),
$$

which clearly satisfies (2.13). The proof is finished.

\subsubsection{Nondegenerate cases}

First we consider a linear perturbation of (7.8) of the form

$$
\begin{aligned}
& x_{1}^{\prime}=\varepsilon\left(a_{0} x_{1}+b_{0} x_{2}+a_{j} x_{1}^{j}+h_{1}(t)\right) \\
& x_{2}^{\prime}=\varepsilon\left(-b_{0} x_{1}+a_{0} x_{2}+a_{j} x_{2}^{j}+h_{2}(t)\right),
\end{aligned}
$$

where $a_{0}, b_{0}, a_{j} \in \mathbb{R}, j \in \mathbb{N}, j \geq 3$ is odd and $h_{1,2}$ satisfy (7.5). Then

$$
J(x, t)=\varepsilon\left(\begin{array}{cc}
a_{0}+j a_{j} x_{1}^{j-1} & 0 \\
0 & a_{0}+j a_{j} x_{2}^{j-1}
\end{array}\right)
$$

which, clearly, satisfies relation (2.12) for $a_{0}<0, a_{j} \leq 0$ and any $\varepsilon>0$. Thus, applying Lemma 2.4, we obtain the following result.

Theorem 7.2. Assume $a_{0}<0, a_{j} \leq 0$. Then nonlinear system (7.12) has the only $2 \pi$-periodic solution $x_{0}(t)=O(\varepsilon)$ for any $\varepsilon>0$, which is moreover $-\pi / 2$ symmetric, i.e., it satisfies (7.11) and it is globally asymptotically stable.

Remark 7.1. Except of Theorem 7.2 in this paper, we do not know anything about dynamics of (1.1) as $\varepsilon$ is increasing.

Finally, to get further interesting results, we consider the following simple form of (7.7):

$$
\begin{aligned}
& f_{1}\left(x_{1}, x_{2}\right)=x_{1}+x_{2}+a x_{1}^{2} x_{2}+b x_{1} x_{2}^{2}, \\
& f_{2}\left(x_{1}, x_{2}\right)=-x_{1}+x_{2}+b x_{1}^{2} x_{2}-a x_{1} x_{2}^{2},
\end{aligned}
$$

where $a, b \in \mathbb{R}$ are constants with $(a, b) \neq(0,0)$. Hence $x_{0}(t)$ is a repeller for $\varepsilon>0$ small. We intend to find more $2 \pi$-periodic solutions of (7.2) with (7.13). For this 
reason, we solve

$$
\begin{array}{r}
x_{1}+x_{2}+a x_{1}^{2} x_{2}+b x_{1} x_{2}^{2}=0 \\
-x_{1}+x_{2}+b x_{1}^{2} x_{2}-a x_{1} x_{2}^{2}=0
\end{array}
$$

which gives

$$
x_{2}\left(2+(a+b) x_{1}^{2}-(a-b) x_{1}\right)=0 .
$$

For $x_{2}=0$ from (7.14) we derive $x_{1}=0$ which gives $x_{0}(t)$. If $x_{2} \neq 0$ and $a=b \geq 0$ then (7.15) has no more solutions. So we suppose $a \neq b$ and then (7.15) gives $x_{1} \neq 0$ along with

$$
x_{2}=\frac{(a+b) x_{2}^{2}+2}{(a-b) x_{1}} .
$$

Inserting (7.16) into the first equation of (7.14), after some computation, we arrive at

$$
2(a+b)+4\left(a^{2}+b^{2}\right) x_{1}^{2}+(a+b)\left(a^{2}+b^{2}\right) x_{1}^{4}=0 .
$$

If $a+b=0$ then (7.17) gives $x_{1}=0$ and so we again obtain $x_{2}=0$. Hence we suppose $a+b \neq 0$, and then (7.17) gives

$$
\begin{aligned}
& x_{1,+}^{2}=\frac{\sqrt{2}(a-b)-2 \sqrt{a^{2}+b^{2}}}{(a+b) \sqrt{a^{2}+b^{2}}}, \\
& x_{1,-}^{2}=-\frac{\sqrt{2}(a-b)+2 \sqrt{a^{2}+b^{2}}}{(a+b) \sqrt{a^{2}+b^{2}}} .
\end{aligned}
$$

It is elementary to observe

$$
\sqrt{2}(a-b)-2 \sqrt{a^{2}+b^{2}}<0, \quad \sqrt{2}(a-b)+2 \sqrt{a^{2}+b^{2}}>0
$$

whenever $a+b \neq 0$. So (7.18) has a real solution if and only if $a+b<0$, which is from now supposed. Then by (7.16), we get four solutions of (7.14). But since according to (7.4), system of equations (7.14) is equivariant with respect to the symmetry (7.3), these solutions are rotationally symmetric (see also Remark 5.1). So we concentrate on the first solution given by the formulae

$$
\begin{aligned}
& x_{1,+}=\sqrt{\frac{\sqrt{2}(a-b)-2 \sqrt{a^{2}+b^{2}}}{(a+b) \sqrt{a^{2}+b^{2}}},} \\
& x_{2,+}=\sqrt{\frac{2(a+b)}{\sqrt{a^{2}+b^{2}}\left(\sqrt{2}(a-b)-2 \sqrt{a^{2}+b^{2}}\right)}} .
\end{aligned}
$$

Then by (7.6) and (7.13) we get

$$
D_{x} P_{1}\left(x_{1,+}, x_{2,+}\right)=\frac{2 \pi}{(a+b) \sqrt{a^{2}+b^{2}}}\left(\begin{array}{cc}
u & v \\
w & w
\end{array}\right),
$$


where

$$
\begin{aligned}
& u=(a-b) \sqrt{a^{2}+b^{2}}+\sqrt{2}\left(2 a^{2}+a b+b^{2}\right), \\
& v=(b-a) \sqrt{a^{2}+b^{2}}+\sqrt{2}\left(a^{2}+a b+2 b^{2}\right),
\end{aligned}
$$

and

$$
w=(a-b) \sqrt{a^{2}+b^{2}}+\sqrt{2}\left(a^{2}+a b+2 b^{2}\right) .
$$

It is interesting to note $\operatorname{det} D_{x} P_{1}\left(x_{1,+}, x_{2,+}\right)=-16 \pi$. The eigenvalues of the matrix $D_{x} P_{1}\left(x_{1,+}, x_{2,+}\right)$ are given by the formulae

$$
\lambda_{ \pm}=2 \pi \frac{a-b \mp \sqrt{9 a^{2}+14 a b+9 b^{2}}}{a+b} .
$$

Since $a+b<0$, we see that $\lambda_{+}>0$ and $\lambda_{-}<0$. Summarizing, we obtain the following result.

Theorem 7.3. Consider the system

$$
\begin{aligned}
& x_{1}^{\prime}=\varepsilon\left(x_{1}+x_{2}+a x_{1}^{2} x_{2}+b x_{1} x_{2}^{2}+h_{1}(t)\right), \\
& x_{2}^{\prime}=\varepsilon\left(-x_{1}+x_{2}+b x_{1}^{2} x_{2}-a x_{1} x_{2}^{2}+h_{2}(t)\right),
\end{aligned}
$$

where $a, b \in \mathbb{R}$ are constants with $(a, b) \neq(0,0), h_{1,2}$ are continuous functions satisfying (7.5) and $\varepsilon>0$ is a small parameter. Then:

(1) If $a+b \geq 0$ then (7.20) has the only $2 \pi$-periodic solution $x_{0}(t)=O(\varepsilon)$ which is in addition a repeller and $-\pi / 2$-symmetric, i. e., it satisfies (7.11).

(2) If $a+b<0$ then (7.20) has in addition precisely four more $2 \pi$-periodic solutions $x_{j}(t), j=1,2,3,4$ which are hyperbolic with the same hyperbolicity type and orbitally $-\pi / 2$-symmetric to each others.

Proof. The proof follows immediately from the above computations together with application of Theorem 5.1 and Remark 5.1.

\section{APPLICATIONS TO PLANAR WEAKLY LINEAR PROBLEMS}

We consider

$$
x^{\prime}=\varepsilon B(t) x,
$$

where $B(t)=\left(B_{i k}(t)\right)_{i, k=1}^{2}$ is a smooth $2 \times 2$-matrix function, $2 \pi$-periodic in $t \in \mathbb{R}$ and $\varepsilon \in \mathbb{R}$ is a small real parameter.

We first study (8.1) without symmetries using standard approach $[8,9,13,19,21$, 22,29]. If $X_{\varepsilon}(t) x=\varphi_{\varepsilon}(x, t)$ is the Cauchy solution of (8.1), where $X_{\varepsilon}(t)$ is its fundamental matrix solution, then we expand $X_{\varepsilon}(t)$ in the form

$$
X_{\varepsilon}(t)=\sum_{i \geq 0} Y_{i}(t) \varepsilon^{i}, \quad Y_{0}(0)=\mathbb{1}, \quad Y_{i}(0)=0 \quad \forall i \geq 1
$$


to derive from (8.1) the recurrence formulas

$$
Y_{i+1}(t)=\int_{0}^{t} B(s) Y_{i}(s) d s, \quad Y_{0}(t)=\mathbb{1} .
$$

Thus, we have

$$
X_{\varepsilon}(2 \pi)=\mathbb{1}+\varepsilon \int_{0}^{2 \pi} B(t) d t+\varepsilon^{2} \int_{0}^{2 \pi} \int_{0}^{t} B(t) B(s) d s d t+O\left(\varepsilon^{3}\right) .
$$

If $\int_{0}^{2 \pi} B(t) d t \neq 0$ then we can apply results of Section 6 (see Remark 6.1). Here we concentrate on the degenerate cases by supposing (see also [9])

$$
\int_{0}^{2 \pi} B(t) d t=0
$$

Then (8.3) becomes

$$
X_{\varepsilon}(2 \pi)=\mathbb{1}+\varepsilon^{2} \int_{0}^{2 \pi} \int_{0}^{t} B(t) B(s) d s d t+O\left(\varepsilon^{3}\right) .
$$

The results of $[19,21,22]$ are difficult to apply to (8.5) (see Remark 6.1). We show that if we consider some symmetries to (8.1), then our theory helps to study such degenerate cases. Now $f(x, t)=B(t) x$, so symmetry condition (1.2) gives

$$
A B(t)=B(t+T) A,
$$

where $A^{p}=\mathbb{1}$ for some $p \in \mathbb{N}, p \geq 2$, and $T=2 \pi / p$. Then we have $X_{\varepsilon}(i T)=$ $A^{i}\left(A^{-1} X_{\varepsilon}(T)\right)^{i}$ for all $i \geq 1$, which implies

$$
\left(A^{-1} X_{\varepsilon}\left(\frac{2 \pi}{p}\right)\right)^{p}=X_{\varepsilon}(2 \pi) \text {. }
$$

We already know (8.7) from Lemma 3.2. In spite of the fact that $Y_{1}(2 \pi)=0$, in many cases we could have $Y_{1}(T) \neq 0$. Thus, using (8.7), we could still study the existence and stability of the zero solution $x=0$ of (8.1) also in the degenerate case (8.4).

Finally, it is better to pass in computations to the complex variable $z=x_{1}+\imath x_{2} \in$ $\mathbb{C}$ for $x=\left(x_{1}, x_{2}\right) \in \mathbb{R}^{2}$. Then (8.1) has the form

$$
\dot{z}=\varepsilon(a(t) z+b(t) \bar{z})
$$

in which

$$
\begin{aligned}
& a(t)=\frac{B_{11}(t)+B_{22}(t)}{2}+\imath \frac{B_{21}(t)-B_{12}(t)}{2}, \\
& b(t)=\frac{B_{11}(t)-B_{22}(t)}{2}+\imath \frac{B_{12}(t)+B_{21}(t)}{2},
\end{aligned}
$$

and $\bar{z}=x_{1}-\imath x_{2}$. Next we again expand

$$
z_{\varepsilon}(t)=\sum_{i \geq 0} w_{i}(t) \varepsilon^{i}, \quad w_{0}(0)=w, \quad w_{i}(0)=0 \quad \forall i \geq 1
$$


to derive from (8.8) the recurrence formulas

$$
w_{i+1}(t)=\int_{0}^{t}\left(a(s) w_{i}(s)+b(s) \bar{w}_{i}(s)\right) d s, \quad w_{0}(t)=w .
$$

In the next sections, we demonstrate the above approach by considering two different classes of symmetries $A$ of (8.1).

\subsection{Rotation symmetries}

We consider the rotation $A z=\mathrm{e}^{\frac{2 \pi}{n}} z$, so $p=n$ and $T=\frac{2 \pi}{n}$. Note $n \in \mathbb{N}$ and $n \geq 3$. Now $f(z, t)=a(t) z+b(t) \bar{z}$, so the symmetry condition (1.2) (see also (8.6)) implies

$$
a(t+T)=a(t), \quad b(t+T)=\mathrm{e}^{\frac{4 \pi}{n} l} b(t) .
$$

Note that (8.4) gives

$$
\int_{0}^{2 \pi} a(t) d t=0, \quad \int_{0}^{2 \pi} b(t) d t=0 .
$$

So both (8.12) and (8.13) imply $\int_{0}^{T} a(t) d t=0$. But we need either $\int_{0}^{T} a(t) d t \neq 0$ or $\int_{0}^{T} b(t) d t \neq 0$. For this reason, we further consider $f(z, t)=b(t) \bar{z}$. Next, expanding $b(t)=\sum_{k \in \mathbb{Z} \backslash\{0\}} b_{k} \mathrm{e}^{k l t}$ and using (8.12) we derive

$$
b(t)=\sum_{m \in \mathbb{Z}} b_{m} \mathrm{e}^{(2+m n) l t} .
$$

Then

$$
\tilde{b}=\int_{0}^{T} b(t) d t=\int_{0}^{2 \pi / n} b(t) d t=\left(\mathrm{e}^{\frac{4 \pi}{n} l}-1\right) \sum_{m \in \mathbb{Z}} \frac{b_{m}}{(2+m n) l} .
$$

In general $\tilde{b} \neq 0$, so by (4.12) we consider the linear map $z \mapsto \tilde{b} \bar{z}$, which for $\tilde{b}=$ $\tilde{b}_{1}+\imath \tilde{b}_{2}$ and $z=x_{1}+\imath x_{2}$ has the form

$$
\left(x_{1}, x_{2}\right) \mapsto\left(\begin{array}{cc}
\widetilde{b}_{1} & \widetilde{b}_{2} \\
\widetilde{b}_{2} & -\widetilde{b}_{1}
\end{array}\right)\left(\begin{array}{l}
x_{1} \\
x_{2}
\end{array}\right) .
$$

However, the matrix $\left(\begin{array}{cc}\widetilde{b}_{1} & \widetilde{b}_{2} \\ \widetilde{b}_{2} & -\widetilde{b}_{1}\end{array}\right) \neq 0$ is always hyperbolic and not asymptotically stable. So we can not apply Theorem 4.1. Consequently, we get now only that the equation

$$
\dot{z}=\varepsilon b(t) \bar{z}
$$


has zero solution the only symmetric one for $\varepsilon \neq 0$ small, when $b(t)$ is given by (8.14). Furthermore, by (8.2) we obtain

$$
\begin{aligned}
A^{-1} X_{\varepsilon}\left(\frac{2 \pi}{n}\right) & =A^{-1}\left(1+\varepsilon Y_{1}\left(\frac{2 \pi}{n}\right)\right)+O\left(\varepsilon^{2}\right) \\
& =\left(\begin{array}{cc}
\cos \frac{2 \pi}{n} & \sin \frac{2 \pi}{n} \\
-\sin \frac{2 \pi}{n} & \cos \frac{2 \pi}{n}
\end{array}\right)\left(\begin{array}{cc}
1+\varepsilon \widetilde{b}_{1} & \varepsilon \widetilde{b}_{2} \\
\varepsilon \widetilde{b}_{2} & 1-\varepsilon \widetilde{b}_{1}
\end{array}\right)+O\left(\varepsilon^{2}\right)
\end{aligned}
$$

The eigenvalues of $A^{-1}\left(\mathbb{1}+\varepsilon Y_{1}\left(\frac{2 \pi}{n}\right)\right)$ are given by

$$
\lambda_{ \pm}=\cos \frac{2 \pi}{n} \pm \frac{l}{\sqrt{2}} \sqrt{1-\cos \frac{4 \pi}{n}-2|\tilde{b}|^{2} \varepsilon^{2}}
$$

for $\varepsilon$ small with

$$
\left|\lambda_{ \pm}\right|=\sqrt{1-|\tilde{b}|^{2} \varepsilon^{2}}=1-\frac{|\widetilde{b}|^{2}}{2} \varepsilon^{2}+O\left(\varepsilon^{3}\right) .
$$

So the matrix $A^{-1}\left(\mathbb{1}+\varepsilon Y_{1}\left(\frac{2 \pi}{n}\right)\right)$ is by Theorem 6.2 strongly 2-hyperbolic, but we should have its strongly 1-hyperbolicity in order to apply results of Section 6 concerning the hyperbolicity of the zero solution. We should need to compute higher order terms in (8.2).

Next, by (8.11) and (8.13), we get

$$
z_{\varepsilon}(2 \pi)=\left(1+\varepsilon^{2} \int_{0}^{2 \pi} \int_{0}^{t} b(t) \bar{b}(s) d s d t\right) w+O\left(\varepsilon^{3}\right),
$$

and using (8.14), we derive

$$
z_{\varepsilon}(2 \pi)=\left(1+\varepsilon^{2} \vartheta_{\imath}\right) w+O\left(\varepsilon^{3}\right)
$$

for

$$
\vartheta=2 \pi \sum_{m \in \mathbb{Z}} \frac{\left|b_{m}\right|^{2}}{2+m n},
$$

which is a nonzero constant for $b(t) \neq 0$. In the variable $x$, we get

$$
X_{\varepsilon}(2 \pi)=B_{2}(\varepsilon)+O\left(\varepsilon^{3}\right)
$$

for

$$
B_{2}(\varepsilon)=\left(\begin{array}{cc}
1 & -\varepsilon^{2} \vartheta \\
\varepsilon^{2} \vartheta & 1
\end{array}\right)=\left(\begin{array}{ll}
1 & 0 \\
0 & 1
\end{array}\right)+\varepsilon^{2}\left(\begin{array}{cc}
0 & -\vartheta \\
\vartheta & 0
\end{array}\right)
$$

Since

$$
1 \notin \sigma\left(\left(\begin{array}{cc}
0 & -\vartheta \\
\vartheta & 0
\end{array}\right)\right)
$$


equation (8.15) has the only zero $2 \pi$-periodic solution. Note the eigenvalues of $B_{2}(\varepsilon)$ are $1 \pm \varepsilon^{2} \vartheta_{l}$. Furthermore, we see that

$$
\left\|B_{2}(\varepsilon)^{-1}\right\|=\frac{1}{\sqrt{1+\varepsilon^{4} \vartheta^{2}}} \leq 1-\frac{\vartheta^{2}}{3} \varepsilon^{4}
$$

for $\varepsilon \neq 0$ small, so by Definition 6.2, $B_{2}(\varepsilon)$ is strongly 4-hyperbolic, but we should have its strongly 2 -hyperbolicity in order to apply results of Section 6 concerning the hyperbolicity of the zero solution. Consequently, the expansion approach (8.2) seems to be difficult for application to (8.15), and hence our approach is as follows. Since $\sigma\left(A^{-1} X_{0}\left(\frac{2 \pi}{n}\right)\right)=\left\{\mathrm{e}^{ \pm 2 \pi l / n}\right\}$, so $\sigma\left(A^{-1} X_{\varepsilon}\left(\frac{2 \pi}{n}\right)\right)=\left\{\lambda_{\varepsilon}, \bar{\lambda}_{\varepsilon}\right\}$ is close to $\left\{\mathrm{e}^{ \pm 2 \pi l / n}\right\}$ for $\varepsilon$ small. Next, (8.15) has the form

$$
\left(\begin{array}{l}
x_{1}^{\prime} \\
x_{2}^{\prime}
\end{array}\right)=\varepsilon\left(\begin{array}{cc}
b_{1}(t) & b_{2}(t) \\
b_{2}(t) & -b_{1}(t)
\end{array}\right)\left(\begin{array}{l}
x_{1} \\
x_{2}
\end{array}\right) .
$$

Hence $\sigma\left(X_{\varepsilon}(2 \pi)\right)=\left\{\lambda_{\varepsilon, 1}, \lambda_{\varepsilon, 2}\right\}$ with $\lambda_{\varepsilon, 1} \lambda_{\varepsilon, 2}=1$. But (8.7) gives $\lambda_{\varepsilon, 1}=\lambda_{\varepsilon}^{p}$ and $\lambda_{\varepsilon, 2}=\bar{\lambda}_{\varepsilon}^{p}=\bar{\lambda}_{\varepsilon, 1}$. This implies that $\left|\lambda_{\varepsilon, 1}\right|=\left|\lambda_{\varepsilon, 2}\right|=1$. Summarizing, we obtain the following result.

Theorem 8.1. Assume (8.13) and $b(t) \neq 0$ is given by (8.14). Then the zero solution is the only symmetric one of equation (8.15) for $\varepsilon \neq 0$ small. It is stable but not asymptotically stable. Moreover, it is the only $2 \pi$-periodic solution of (8.15).

We see that simple arguments using symmetries of (8.15) provide the stability of this equation, while expansion method seems to be rather awkward.

\subsection{Reflection symmetries}

Now we consider $A z=\mathrm{e}^{\frac{\pi}{n} l} \bar{z}$ with $p=2 n$ and $T=\frac{\pi}{n}$. Note that $A$ has the form

$$
A=\left(\begin{array}{cc}
\cos \frac{\pi}{n} & -\sin \frac{\pi}{n} \\
-\sin \frac{\pi}{n} & -\cos \frac{\pi}{n}
\end{array}\right),
$$

satisfies the relation $A^{2}=\mathbb{1}$, and has the eigenvalues 1 and -1 and the corresponding orthogonal eigenvectors $\left(\sin \frac{\pi}{n}, \cos \frac{\pi}{n}-1\right)$ and $\left(\sin \frac{\pi}{n}, 1+\cos \frac{\pi}{n}\right)$, respectively. Thus, $A$ is the reflection with respect to the line $x_{2}=-\tan \left(\frac{\pi}{2 n}\right) x_{1}$.

Next, the symmetry condition (1.2) (see also (8.6)) gives

$$
a(t+T)=\bar{a}(t), \quad b(t+T)=\mathrm{e}^{\frac{2 \pi}{n} l} \bar{b}(t) .
$$

Using the above approach, we derive

$$
a(t)=\sum_{k \in \mathbb{N}}\left(a_{k}+\bar{a}_{k} \mathrm{e}^{k \frac{\pi}{n} l}\right) \mathrm{e}^{k t l}
$$

and

$$
b(t)=\sum_{k \in \mathbb{N}}\left(b_{k}+\bar{b}_{k} \mathrm{e}^{(2+k) \frac{\pi}{n} l}\right) \mathrm{e}^{k t l} .
$$


Then

$$
\widehat{a}=\int_{0}^{T} a(t) d t=\int_{0}^{\pi / n} a(t) d t=\sum_{k \in \mathbb{N}}\left(a_{k}+\bar{a}_{k} \mathrm{e}^{k \frac{\pi}{n} l}\right) \frac{\mathrm{e}^{k \frac{\pi}{n} l}-1}{k \imath}
$$

and

$$
\widehat{b}=\int_{0}^{T} b(t) d t=\int_{0}^{\pi / n} b(t) d t=\sum_{k \in \mathbb{N}}\left(b_{k}+\bar{b}_{k} \mathrm{e}^{(2+k) \frac{\pi}{n} l}\right) \frac{\mathrm{e}^{k \frac{\pi}{n} l}-1}{k l} .
$$

In general $\hat{a} \neq 0$ and $\hat{b} \neq 0$, so by (4.12) we consider the linear map $z \mapsto \hat{a} z+\hat{b} \bar{z}$.

Now we want to show that for any given $\hat{a}, \hat{b} \in \mathbb{C}$, we can find $a(t), b(t)$ satisfying (8.20) and (8.21). For this reason, we first derive

$$
\begin{aligned}
\left(a_{k}+\bar{a}_{k} \mathrm{e}^{k \frac{\pi}{n} l}\right) \frac{\mathrm{e}^{k \frac{\pi}{n} l}-1}{k l} & =\left(a_{k} \mathrm{e}^{-k \frac{\pi}{2 n} l}+\bar{a}_{k} \mathrm{e}^{k \frac{\pi}{2 n} l}\right) \mathrm{e}^{k \frac{\pi}{n} l} \frac{\mathrm{e}^{k \frac{\pi}{2 n} l}-\mathrm{e}^{-k \frac{\pi}{2 n} l}}{k l} \\
& =\left(4 \operatorname{Re}\left\{a_{k} \mathrm{e}^{-k \frac{\pi}{2 n} l}\right\} \frac{\sin k \frac{\pi}{2 n}}{k}\right) \mathrm{e}^{k \frac{\pi}{n} l}
\end{aligned}
$$

So the range of the mapping

$$
\mathbb{C} \ni a_{k} \mapsto\left(a_{k}+\bar{a}_{k} \mathrm{e}^{k \frac{\pi}{n} l}\right) \frac{\mathrm{e}^{k \frac{\pi}{n} l}-1}{k l} \in \mathbb{C} \sim \mathbb{R}^{2}
$$

in the plain $\mathbb{R}^{2}$ is the line $x_{2}=\tan k \frac{\pi}{n} x_{1}$. Consequently, the mapping

$$
\mathbb{C} \times \mathbb{C} \ni\left(a_{1}, a_{2}\right) \mapsto\left(a_{1}+\bar{a}_{1} \mathrm{e}^{\frac{\pi}{n} l}\right) \frac{\mathrm{e}^{\frac{\pi}{n} l}-1}{l}+\left(a_{2}+\bar{a}_{2} \mathrm{e}^{2 \frac{\pi}{n} l}\right) \frac{\mathrm{e}^{2 \frac{\pi}{n} l}-1}{2 l} \in \mathbb{C}
$$

is onto (surjective). Hence, for any $\hat{a} \in \mathbb{C}$, there are $a_{1}, a_{2} \in \mathbb{C}$ such that (8.20) holds with

$$
a(t)=\left(a_{1}+\bar{a}_{1} \mathrm{e}^{\frac{\pi}{n} l}\right) \mathrm{e}^{t \imath}+\left(a_{2}+\bar{a}_{2} \mathrm{e}^{2 \frac{\pi}{n} l}\right) \mathrm{e}^{2 t l} .
$$

Similarly we have

$$
\left(b_{k}+\bar{b}_{k} \mathrm{e}^{(2+k) \frac{\pi}{n} l}\right) \frac{\mathrm{e}^{k \frac{\pi}{n} l}-1}{k l}=\left(4 \operatorname{Re}\left\{b_{k} \mathrm{e}^{-(2+k) \frac{\pi}{2 n} l}\right\} \frac{\sin k \frac{\pi}{2 n}}{k}\right) \mathrm{e}^{(1+k) \frac{\pi}{n} l} .
$$

So the range of the mapping

$$
\mathbb{C} \ni b_{k} \mapsto\left(b_{k}+\bar{b}_{k} \mathrm{e}^{(2+k) \frac{\pi}{n} l}\right) \frac{\mathrm{e}^{k \frac{\pi}{n} l}-1}{k l} \in \mathbb{C} \sim \mathbb{R}^{2}
$$

in the plain $\mathbb{R}^{2}$ is the line $x_{2}=\tan (1+k) \frac{\pi}{n} x_{1}$. Consequently, the mapping

$$
\mathbb{C} \times \mathbb{C} \ni\left(b_{1}, b_{2}\right) \mapsto\left(b_{1}+\bar{b}_{1} \mathrm{e}^{3 \frac{\pi}{n} l}\right) \frac{\mathrm{e}^{\frac{\pi}{n} l}-1}{l}+\left(b_{2}+\bar{b}_{2} \mathrm{e}^{4 \frac{\pi}{n} l}\right) \frac{\mathrm{e}^{2 \frac{\pi}{n} l}-1}{2 l} \in \mathbb{C}
$$


is onto (surjective). Hence, for any $\hat{b} \in \mathbb{C}$, there are $b_{1}, b_{2} \in \mathbb{C}$ such that (8.21) holds with

$$
b(t)=\left(b_{1}+\bar{b}_{1} \mathrm{e}^{3 \frac{\pi}{n} l}\right) \mathrm{e}^{t l}+\left(b_{2}+\bar{b}_{2} \mathrm{e}^{4 \frac{\pi}{n} l}\right) \mathrm{e}^{2 t l} .
$$

Summarizing we see that, for any given $a, b, c, d \in \mathbb{R}$, by adjusting suitable $a_{1}, a_{2}, b_{1}, b_{2} \in$ $\mathbb{C}$, we have $Y_{1}\left(\frac{\pi}{n}\right)=\left(\begin{array}{ll}a & b \\ c & d\end{array}\right)$. Next we derive

$$
\begin{aligned}
A^{-1} X_{\varepsilon}\left(\frac{\pi}{n}\right) & =A^{-1}\left(\mathbb{1}+\varepsilon Y_{1}\left(\frac{\pi}{n}\right)\right)+O\left(\varepsilon^{2}\right) \\
& =\left(\begin{array}{cc}
\cos \frac{\pi}{n} & -\sin \frac{\pi}{n} \\
-\sin \frac{\pi}{n} & -\cos \frac{\pi}{n}
\end{array}\right)\left(\begin{array}{cc}
1+\varepsilon a & \varepsilon b \\
\varepsilon c & 1+\varepsilon d
\end{array}\right)+O\left(\varepsilon^{2}\right) .
\end{aligned}
$$

The eigenvalues $\tilde{\lambda}_{ \pm}$of $A^{-1}\left(\mathbb{1}+\varepsilon Y_{1}\left(\frac{\pi}{n}\right)\right)$ are given by the equalities

$$
\begin{aligned}
\tilde{\lambda}_{ \pm}= & \frac{1}{2}\left(\varepsilon(a-d) \cos \frac{\pi}{n}-\varepsilon(b+c) \sin \frac{\pi}{n}\right. \\
& \left. \pm \sqrt{4+4 \varepsilon(a+d)+\varepsilon^{2}\left(a d-b c+\left((d-a) \cos \frac{\pi}{n}+(b+c) \sin \frac{\pi}{n}\right)^{2}\right)}\right)
\end{aligned}
$$

for $\varepsilon$ small, which yield

$$
\left.\begin{array}{l}
\tilde{\lambda}_{+}=1+\varepsilon \frac{\sin \frac{\pi}{n}}{2}\left(a \cot \frac{\pi}{2 n}+d \tan \frac{\pi}{2 n}-(b+c)\right)+O\left(\varepsilon^{2}\right), \\
\tilde{\lambda}_{-}=-1-\varepsilon \frac{\sin \frac{\pi}{n}}{2}\left(a \tan \frac{\pi}{2 n}+d \cot \frac{\pi}{2 n}+(b+c)\right)+O\left(\varepsilon^{2}\right) .
\end{array}\right\}
$$

The two planes

$$
\begin{aligned}
& x_{1} \cot \frac{\pi}{2 n}+x_{2} \tan \frac{\pi}{2 n}-x_{3}=0, \\
& x_{1} \tan \frac{\pi}{2 n}+x_{2} \cot \frac{\pi}{2 n}+x_{3}=0
\end{aligned}
$$

decompose the space $\mathbb{R}^{3}$ into four subspaces where, by (8.24), we get four different types of a strong 1-hyperbolicity of $A^{-1}\left(\mathbb{1}+\varepsilon Y_{1}\left(\frac{\pi}{n}\right)\right)$ with the corresponding hyperbolicities of $A^{-1} X_{\varepsilon}\left(\frac{\pi}{n}\right)$. For instance, $A^{-1} X_{\varepsilon}\left(\frac{\pi}{n}\right)$ is asymptotically stable for $\varepsilon>0$ small if

$$
a \cot \frac{\pi}{2 n}+d \tan \frac{\pi}{2 n}<b+c<-a \tan \frac{\pi}{2 n}-d \cot \frac{\pi}{2 n} \text {. }
$$

Note that (8.25) yields $a+d<0$. On the other hand, the real parts of the eigenvalues of $\left(\begin{array}{ll}a & b \\ c & d\end{array}\right)$ lie in $\subset(-\infty, 0)$ if and only if

$$
a+d<0, \quad a d-b c>0,
$$

which condition follows from the Routh-Hurwitz criterion [6]. The relationship between constants $a, b, c, d \in \mathbb{R}$ and $a_{1}, a_{2}, b_{1}, b_{2} \in \mathbb{C}$ are given by (8.20), (8.21) and 
(see (8.9)) also by the equalities

$$
a=\operatorname{Re} \hat{a}+\operatorname{Re} \hat{b}, \quad b=\operatorname{Im} \hat{b}-\operatorname{Im} \hat{a}, \quad c=\operatorname{Im} \hat{a}+\operatorname{Im} \hat{b}, \quad d=\operatorname{Re} \hat{a}-\operatorname{Re} \hat{b},
$$

which are obtained by solving the equality

$$
\widehat{a} z+\hat{b} \bar{z}=\left(\begin{array}{ll}
a & b \\
c & d
\end{array}\right)\left(\begin{array}{l}
x_{1} \\
x_{2}
\end{array}\right),
$$

where $z=x_{1}+\imath x_{2}$, for any $x_{1}$ and $x_{2}$ from $\mathbb{R}$. Inserting (8.27) into (8.25) and (8.26), summarizing, we obtain the following result.

Theorem 8.2. Assume (8.13), and $a(t)$ and $b(t)$ are given by (8.22) and (8.23), respectively. Then the equation

$$
\dot{z}=\varepsilon(a(t) z+b(t) \bar{z})
$$

has zero solution the only symmetric one for $\varepsilon \neq 0$ small which can be either hyperbolic, asymptotically stable or unstable, respectively. ${ }^{\dagger}$ For instance, it is asymptotically stable for $\varepsilon>0$ small if $\operatorname{Re} \hat{a}<0$ and either $|\widehat{a}|>|\widehat{b}|$ or the inequalities

$$
\operatorname{Re} \hat{a} \sec \frac{\pi}{n}+\operatorname{Re} \hat{b} \cot \frac{\pi}{n}<\operatorname{Im} \hat{b}<-\operatorname{Re} \hat{a} \sec \frac{\pi}{n}+\operatorname{Re} \hat{b} \cot \frac{\pi}{n}
$$

are satisfied. Constants $\hat{a}$ and $\hat{b}$ are given by (8.20) and (8.21), respectively.

We do not compute $Y_{2}(2 \pi)$ in (8.2) for (8.28) since it is an awkward formula, but we again see that in general, for instance in the hyperbolic cases, the zero solution is the only $2 \pi$-periodic solution of (8.28).

\section{REFERENCES}

[1] S. Aizicovici and M. Fečkan, "Forced symmetric oscillations of evolution equations," Nonlinear Anal., vol. 64, no. 7, pp. 1621-1640, 2006.

[2] S. Aizicovici and N. H. Pavel, "Anti-periodic solutions to a class of nonlinear differential equations in Hilbert space," J. Funct. Anal., vol. 99, no. 2, pp. 387-408, 1991.

[3] B. F. Bylov, R. È. Vinograd, D. M. Grobman, and V. V. Nemyckiŭ, Teoriya pokazatelei Lyapunova i ee prilozheniya $k$ voprosam ustoichivosti. Izdat. "Nauka", Moscow, 1966.

[4] W. A. Coppel, Stability and asymptotic behavior of differential equations. D. C. Heath and Co., Boston, Mass., 1965.

[5] Mitropol'skiü, Yu. A. and J. A. Mitropol'skiǔ, Metod usredneniya v nelineinoi mekhanike. Kiev: "Naukova Dumka", 1971.

[6] B. P. Demidovič, Lektsii po matematicheskoi teorii ustoichivosti. Izdat. "Nauka", Moscow, 1967.

[7] M. Fečkan, R. Ma, and B. Thompson, "Forced symmetric oscillations," Bull. Belg. Math. Soc. Simon Stevin, vol. 14, no. 1, pp. 73-85, 2007.

[8] J. Guckenheimer and P. Holmes, Nonlinear oscillations, dynamical systems, and bifurcations of vector fields, ser. Applied Mathematical Sciences. New York: Springer-Verlag, 1983, vol. 42.

[9] Hartono and A. H. P. van der Burgh, "Higher-order averaging: periodic solutions, linear systems and an application," Nonlinear Anal., vol. 52, no. 7, pp. 1727-1744, 2003.

\footnotetext{
${ }^{\dagger}$ Of course, depending on the choice of $a_{1}, a_{2}, b_{1}, b_{2} \in \mathbb{C}$.
} 
[10] M. W. Hirsch and S. Smale, Differential equations, dynamical systems, and linear algebra. Academic Press [A subsidiary of Harcourt Brace Jovanovich, Publishers], New York-London, 1974, pure and Applied Mathematics, Vol. 60.

[11] M. C. Irwin, Smooth dynamical systems, ser. Pure and Applied Mathematics. New York: Academic Press Inc. [Harcourt Brace Jovanovich Publishers], 1980, vol. 94.

[12] V. Lakshmikantham, S. Leela, and A. A. Martynyuk, Practical stability of nonlinear systems. Teaneck, NJ: World Scientific Publishing Co. Inc., 1990.

[13] M. Levi, "Geometry and physics of averaging with applications," Phys. D, vol. 132, no. 1-2, pp. 150-164, 1999.

[14] M. Y. Li and J. S. Muldowney, "A geometric approach to global-stability problems," SIAM J. Math. Anal., vol. 27, no. 4, pp. 1070-1083, 1996.

[15] M. Y. Li and J. S. Muldowney, "Phase asymptotic semiflows, Poincaré's condition, and the existence of stable limit cycles," J. Differential Equations, vol. 124, no. 2, pp. 425-448, 1996.

[16] S. M. Lozinskiŭ, "Error estimate for numerical integration of ordinary differential equations. I," Izv. Vyš̌. Učebn. Zaved. Matematika, no. 5 (6), pp. 52-90, 1958.

[17] M. Medved', Fundamentals of dynamical systems and bifurcation theory. Bristol: Adam Hilger Ltd., 1992.

[18] J. A. Mitropol'skiı̌ and V. I. Fodčuk, "The second theorem of N. N. Bogoljubov on the averaging method for differential equations with retarded argument," Ukrain. Mat. Ž., vol. 24, pp. 49-56, 1972.

[19] J. T. Montgomery, "Existence and stability of periodic motion under higher order averaging," $J$. Differential Equations, vol. 64, no. 1, pp. 67-78, 1986.

[20] F. J. Muñoz-Almaraz, E. Freire, J. Galan-Vioque, and A. Vanderbauwhede, "Continuation of normal doubly symmetric orbits in conservative reversible systems," Celestial Mech. Dynam. Astronom., vol. 97, no. 1, pp. 17-47, 2007.

[21] J. Murdock, "Qualitative theory of nonlinear resonance by averaging and dynamical systems methods," in Dynamics reported, Vol. 1, ser. Dynam. Report. Ser. Dynam. Systems Appl. Chichester: Wiley, 1988, vol. 1, pp. 91-172.

[22] J. Murdock and C. Robinson, "Qualitative dynamics from asymptotic expansions: local theory," J. Differential Equations, vol. 36, no. 3, pp. 425-441, 1980.

[23] A. Rontó and M. Rontó, "On some symmetric properties of periodic solutions," Nonlinear Oscil., vol. 6, no. 1, pp. 82-107, 2003.

[24] A. Rontó and M. Rontó, Handbook of Differential Equations: Ordinary Differential Equations. Amsterdam: Elsevier/North-Holland, 2008, vol. IV, ch. 5. Successive Approximation Techniques in Non-Linear Boundary Value Problems for Ordinary Differential Equations, pp. 441-592.

[25] W. Rudin, Real and complex analysis, 2nd ed. New York: McGraw-Hill Book Co., 1974, McGraw-Hill Series in Higher Mathematics.

[26] A. M. Samoullenko and R. I. Petrishin, "The averaging method in multifrequency systems with slowly varying parameters," Ukrain. Math. J., vol. 40, no. 4, pp. 425-431, 1988.

[27] J. A. Sanders and F. Verhulst, Averaging methods in nonlinear dynamical systems, ser. Applied Mathematical Sciences. New York: Springer-Verlag, 1985, vol. 59.

[28] A. R. Teel, J. Peuteman, and D. Aeyels, "Semi-global practical asymptotic stability and averaging," Systems Control Lett., vol. 37, no. 5, pp. 329-334, 1999.

[29] V. A. Yakubovich and V. M. Starzhinskii, Linear differential equations with periodic coefficients. 1, 2. Halsted Press [John Wiley \& Sons] New York-Toronto, Ont.,, 1975, translated from Russian by D. Louvish.

[30] T. Yoshizawa, Stability theory and the existence of periodic solutions and almost periodic solutions. New York: Springer-Verlag, 1975, applied Mathematical Sciences, Vol. 14. 
Authors' addresses

Nataliya Dilna

Institute of Mathematics of the National Academy of Sciences of Ukraine, 3 Tereshchenkivska St., 01601 Kiev, Ukraine

Current address: Mathematical Institute of the Slovak Academy of Sciences, Štefánikova 49, Bratislava, Slovak Republic

E-mail address: dilna@imath.kiev.ua

Michal Fečkan

Department of Mathematical Analysis and Numerical Mathematics, Comenius University, Mlynská dolina, 84248 Bratislava, Slovak Republic

Mathematical Institute of Slovak Academy of Sciences, Štefánikova 49, Bratislava, Slovak Republic

E-mail address: Michal.Feckan@fmph.uniba.sk 